\title{
ON THE STABILITY OF BRESSE AND TIMOSHENKO SYSTEMS WITH HYPERBOLIC HEAT CONDUCTION
}

\author{
FILIPPO DELL'ORO
}

\begin{abstract}
We investigate the stability of three thermoelastic beam systems with hyperbolic heat conduction. First, we study the Bresse-Gurtin-Pipkin system, providing a necessary and sufficient condition for the exponential stability and the optimal polynomial decay rate when the condition is violated. Second, we obtain analogous results for the Bresse-Maxwell-Cattaneo system, completing an analysis recently initiated in the literature. Finally, we consider the Timoshenko-Gurtin-Pipkin system and we find the optimal polynomial decay rate when the known exponential stability condition does not hold. As a byproduct, we fully recover the stability characterization of the Timoshenko-Maxwell-Cattaneo system. The classical "equal wave speeds" conditions are also recovered through singular limit procedures. Our conditions are compatible with some physical constraints on the coefficients as the positivity of the Poisson's ratio of the material. The analysis faces several challenges connected with the thermal damping, whose resolution rests on recently developed mathematical tools such as quantitative RiemannLebesgue lemmas.
\end{abstract}

\section{INTRODUCTION}

1.1. Preamble. In 1993-1994, J.E. Lagnese, G. Leugering and E.J.P.G. Schmidt derived a general nonlinear PDE model for the dynamics of thin thermoelastic beams [30, 31]. A particular linearized case of such a model is the Bresse-Fourier (BF) system

$$
\left\{\begin{array}{l}
\rho_{1} \varphi_{t t}-k\left(\varphi_{x}+\psi+l w\right)_{x}-l k_{0}\left(w_{x}-l \varphi\right)+l \gamma \xi=0, \\
\rho_{2} \psi_{t t}-b \psi_{x x}+k\left(\varphi_{x}+\psi+l w\right)+\gamma \vartheta_{x}=0, \\
\rho_{1} w_{t t}-k_{0}\left(w_{x}-l \varphi\right)_{x}+l k\left(\varphi_{x}+\psi+l w\right)+\gamma \xi_{x}=0, \\
\rho_{3} \vartheta_{t}-\varpi \vartheta_{x x}+\gamma \psi_{x t}=0, \\
\rho_{3} \xi_{t}-\varpi \xi_{x x}+\gamma\left(w_{x t}-l \varphi_{t}\right)=0,
\end{array}\right.
$$

which describes the vibrations of a curved thin thermoelastic beam of length $\ell>0$, taking into account both rotatory inertia and shear deformation effects. The unknowns $\varphi, \psi, w$ represent the vertical displacement, the rotation angle of the cross-section and the horizontal displacement, while $\vartheta, \xi$ represent the temperature (deviations from a fixed reference temperature) along the vertical and horizontal directions. With standard notation, the subscripts $t$ and $x$ indicate the partial derivatives with respect to the time variable $t>0$ and the space variable $x \in(0, \ell)$. The strictly positive constants $\rho_{1}, \rho_{2}, \rho_{3}, k, k_{0}, b, \varpi, l, \gamma$ account for the physical properties of

2010 Mathematics Subject Classification. 35B40, 45K05, 47D03, 74D05, 74F05.

Key words and phrases. Bresse system, Timoshenko system, Gurtin-Pipkin law, Maxwell-Cattaneo law, exponential stability, polynomial stability. 
the beam (see $[30,31,32]$ for details). In particular, they are subjected to the constraints

$$
k_{0}=\frac{b \rho_{1}}{\rho_{2}} \quad \text { and } \quad b>\frac{k \rho_{2}}{\rho_{1}} .
$$

The first equality tells that the rotation angle and the horizontal displacement motions have the same wave speeds. The second relation tells that the wave speed of the rotation angle equation is greater than the one of the vertical displacement equation, and is a consequence of the positivity of the Poisson's ratio of the involved material (which is always the case for "ordinary" media). Finally, the constant $l$ accounts for the initial curvature of the beam. However, as customary in the mathematical literature on the subject, in the sequel we will unhook these constants from their physical meaning, allowing them to assume any positive real value. Still, we will keep in mind (1.2) as it permits a physical interpretation of the mathematical results.

Remark 1.1. System (1.1) takes the first part of the name (Bresse) from the fact that its isothermal counterpart (i.e. when the temperatures are neglected) was derived in 1859 by the French engineer J.A.C. Bresse in his pioneering work [5]. The second part of the name (Fourier) is because the temperature evolution is modeled using the Fourier law (see $[30,31]$ for details).

It is interesting to observe that in the limit case when $l=0$, namely when the beam is straight, the horizontal displacement uncouples from the vertical and the rotation angle motions. In this situation (1.1) splits into the Timoshenko-Fourier (TF) system

$$
\left\{\begin{array}{l}
\rho_{1} \varphi_{t t}-k\left(\varphi_{x}+\psi\right)_{x}=0, \\
\rho_{2} \psi_{t t}-b \psi_{x x}+k\left(\varphi_{x}+\psi\right)+\gamma \vartheta_{x}=0, \\
\rho_{3} \vartheta_{t}-\varpi \vartheta_{x x}+\gamma \psi_{x t}=0,
\end{array}\right.
$$

and the system of second-order linear thermoelasticity in one dimension

$$
\left\{\begin{array}{l}
\rho_{1} w_{t t}-k_{0} w_{x x}+\gamma \xi_{x}=0, \\
\rho_{3} \xi_{t}-\varpi \xi_{x x}+\gamma w_{x t}=0 .
\end{array}\right.
$$

Therefore, one may assert that (1.3) and (1.4) are both special cases of (1.1). Of course, since the constant $l$ is assumed to be strictly positive, one should more properly say that (1.3) and (1.4) can be obtained from (1.1) in the (singular) limit $l \rightarrow 0$.

Remark 1.2. System (1.3) takes the first part of the name from the fact that its isothermal counterpart is the well-known Timoshenko system [50] or, more properly, the TimoshenkoEhrenfest system (see the historical account [20]).

1.2. Stability of the BF and TF systems. The stability properties of the BF system (1.1) have been analyzed for the first time by Z. Liu and B. Rao in the influential paper [32]. There, the authors introduced two stability numbers

$$
\chi_{0}=b-\frac{k \rho_{2}}{\rho_{1}} \quad \text { and } \quad \chi_{1}=k_{0}-k,
$$

and showed that the solution semigroup associated to (1.1) with appropriate boundary conditions is exponentially stable if and only if

$$
\chi_{0} \chi_{1}=0 .
$$


This means that exponential stability occurs if and only if the first and the second equation of (1.1) have the same wave speed or the same do the first and the third equation. When (1.5) is violated, it was proved in [32] that the semigroup is polynomially stable. Note that, since (1.2) is incompatible with (1.5), exponential stability never occurs in physical situations.

Concerning the TF system (1.3), it was proved by J.E. Muñoz Rivera and R. Racke [37] that the associated solution semigroup is exponentially stable if and only if $\chi_{0}=0$. Again, the latter condition is physically unrealistic [38] and, when violated, leads to a polynomially stable dynamics [6].

Remark 1.3. It is a classical result that the semigroup associated to the system of secondorder thermoelasticity (1.4) is exponentially stable independently of the value of the structural constants in the model (see e.g. [33, Chapter 2] and [44, Chapter 2]).

The meaning of (1.5) has been highlighted in [32], where it is explained that the variables $\psi$ and $w$ are "effectively damped" due to the coupling with the temperatures but the variable $\varphi$ is only "indirectly damped" via the second and third equations plus a weaker coupling with the temperature. Condition (1.5) tells that the effectiveness of the damping acting on $\varphi$ depends on the equality between the wave speeds of either the second (effectively damped) equation and the first equation, or the third (effectively damped) equation and the first equation. From the technical side, condition (1.5) provides a cancellation of some higher-order terms that cannot be controlled with the first-order energy. A similar phenomenon appears in the TF system.

1.3. Hyperbolic heat conduction. In the physical derivation of system (1.1) one employs the classical Fourier thermal law

$$
p=-\varpi \vartheta_{x},
$$

where $p=p(x, t)$ is the so-called heat-flux variable (see $[30,31]$ for details). But, as is wellknown, the use of (1.6) leads to an infinite speed propagation of thermal signals, due to the parabolic character of the heat equation. On the contrary, with the advent of modern microscale technologies, there is an increasing evidence that the thermal motion is a wave-type phenomenon, where the temperature may travel with a finite speed of propagation (see e.g. [49] and references therein). As a consequence, a number of "hyperbolic heat conduction theories" have been proposed along the years. One of them is due to M.E. Gurtin and A.C. Pipkin [28] and consists in replacing (1.6) with

$$
p(t)=-\varpi \int_{0}^{\infty} g(s) \vartheta_{x}(t-s) \mathrm{d} s,
$$

where $g$ is a suitable convolution kernel. Equation (1.7) is a memory relaxation of (1.6), and the latter can be recovered in the (singular) limit when $g$ converges to the Dirac mass at zero. An interesting special case of (1.7) is obtained by choosing

$$
g(s)=\frac{1}{\varpi \varsigma} \mathrm{e}^{-\frac{s}{\varpi \varsigma}}
$$

where $\varsigma$ is a positive parameter. In this way, one gets from (1.7) the so-called Maxwell-Cattaneo law [7], that is

$$
\varsigma \varpi p_{t}+p=-\varpi \vartheta_{x} .
$$

Note that (1.8) reduces to (1.6) in the limit situation when $\varsigma=0$. 
On the basis of these motivations, there has been a lot of activity directed towards the study of PDE models where the parabolic Fourier law is replaced by a hyperbolic one. To the best of our knowledge, the first results concerning the stability properties of Bresse and Timoshenko systems with hyperbolic heat conduction have been obtained in the influential papers [15, 46], dealing with the thermoelastic Timoshenko system with temperature obeying the Maxwell-Cattaneo law. The analysis has been extended to the Timoshenko system with Gurtin-Pipkin law in [19]. More recently, the Bresse system with Maxwell-Cattaneo law has been studied in [17]. Further papers treating "reduced Bresse models" where one temperature is neglected have appeared in the literature (see Section 2 for details), in addition to other articles where different damping mechanisms are analyzed. The number of contributions on the subject is rather big, and a comprehensive overview looks prohibitive. Disregarding papers dealing exclusively with the Timoshenko system, we may cite $[1,8,21,22,23,26,27,29,34,35,36,45,47,48,51]$, but the list is not exhaustive. Still, to the best of our knowledge, the stability properties of the Bresse system with Gurtin-Pipkin law have not been investigated so far. Moreover, the analyses in $[17,19]$ only deal with the exponential stability, and no decay rates have been established when exponential stability does not occur. The aim of the present paper is to fill these gaps.

1.4. Main results. We now describe briefly and informally our main results, postponing the rigorous statements to the forthcoming Sections 4-6.

I. First, we study the Bresse-Gurtin-Pipkin (BGP) system

$$
\left\{\begin{array}{l}
\rho_{1} \varphi_{t t}-k\left(\varphi_{x}+\psi+l w\right)_{x}-l k_{0}\left(w_{x}-l \varphi\right)+l \gamma \xi=0, \\
\rho_{2} \psi_{t t}-b \psi_{x x}+k\left(\varphi_{x}+\psi+l w\right)+\gamma \vartheta_{x}=0, \\
\rho_{1} w_{t t}-k_{0}\left(w_{x}-l \varphi\right)_{x}+l k\left(\varphi_{x}+\psi+l w\right)+\gamma \xi_{x}=0, \\
\rho_{3} \vartheta_{t}-\varpi \int_{0}^{\infty} g(s) \vartheta_{x x}(t-s) \mathrm{d} s+\gamma \psi_{x t}=0, \\
\rho_{3} \xi_{t}-\varpi \int_{0}^{\infty} h(s) \xi_{x x}(t-s) \mathrm{d} s+\gamma\left(w_{x t}-l \varphi_{t}\right)=0 .
\end{array}\right.
$$

The model is complemented with the Dirichlet boundary conditions for the variables $\varphi, \vartheta, \xi$

$$
\varphi(0, t)=\varphi(\ell, t)=\vartheta(0, t)=\vartheta(\ell, t)=\xi(0, t)=\xi(\ell, t)=0,
$$

the Neumann boundary conditions for the variables $\psi, w$

$$
\psi_{x}(0, t)=\psi_{x}(\ell, t)=w_{x}(0, t)=w_{x}(\ell, t)=0,
$$

and the appropriate initial data. In particular, $\vartheta$ and $\xi$ are supposed to be known for negative times, where they need not solve the equations. The convolution kernels $g$ and $h$ are nonnegative bounded convex summable functions on $[0, \infty)$, both of unitary total mass and subjected to some additional properties that will be specified in Subsection 4.1. Exploiting the so-called history framework devised by C.M. Dafermos [12], system (1.9) can be shown to generate a solution semigroup $S(t)$ on an appropriate phase space. Introducing the two stability numbers

$$
\chi_{g}=\left(\frac{\rho_{3}}{\varpi g(0)}-\frac{\rho_{1}}{k}\right)\left(b-\frac{k \rho_{2}}{\rho_{1}}\right)+\frac{\gamma^{2}}{\varpi g(0)},
$$




$$
\chi_{h}=\left(\frac{\rho_{3}}{\varpi h(0)}-\frac{\rho_{1}}{k}\right)\left(k_{0}-k\right)+\frac{\gamma^{2}}{\varpi h(0)},
$$

we prove that the semigroup $S(t)$ is exponentially stable if and only if

$$
\chi_{g} \chi_{h}=0 \text {. }
$$

Moreover, when $\chi_{g} \chi_{h} \neq 0$, we show that $S(t)$ is (semiuniformly) polynomially stable with optimal decay rate $\sqrt{t}$.

II. Second, we analyze the Bresse-Maxwell-Cattaneo (BMC) system

$$
\left\{\begin{array}{l}
\rho_{1} \varphi_{t t}-k\left(\varphi_{x}+\psi+l w\right)_{x}-l k_{0}\left(w_{x}-l \varphi\right)+l \gamma \xi=0, \\
\rho_{2} \psi_{t t}-b \psi_{x x}+k\left(\varphi_{x}+\psi+l w\right)+\gamma \vartheta_{x}=0, \\
\rho_{1} w_{t t}-k_{0}\left(w_{x}-l \varphi\right)_{x}+l k\left(\varphi_{x}+\psi+l w\right)+\gamma \xi_{x}=0, \\
\rho_{3} \vartheta_{t}+p_{x}+\gamma \psi_{x t}=0, \\
\varsigma \varpi p_{t}+p+\varpi \vartheta_{x}=0, \\
\rho_{3} \xi_{t}+q_{x}+\gamma\left(w_{x t}-l \varphi_{t}\right)=0, \\
\tau \varpi q_{t}+q+\varpi \xi_{x}=0,
\end{array}\right.
$$

complemented with the boundary conditions (1.10)-(1.11) and the appropriate initial data. The unknowns $p$ and $q$ represent the heat-flux variables, and the constants $\varsigma$ and $\tau$ are strictly positive. In [17], the BMC system (1.13) is shown to generate a solution semigroup $T(t)$ on the natural phase space. In the same article, the authors introduced the stability numbers

$$
\chi_{\varsigma}=\left(\varsigma \rho_{3}-\frac{\rho_{1}}{k}\right)\left(b-\frac{k \rho_{2}}{\rho_{1}}\right)+\gamma^{2} \varsigma \quad \text { and } \quad \chi_{\tau}=\left(\tau \rho_{3}-\frac{\rho_{1}}{k}\right)\left(k_{0}-k\right)+\gamma^{2} \tau,
$$

and proved that the semigroup $T(t)$ is exponentially stable when

$$
\chi_{\varsigma} \chi_{\tau}=0 .
$$

Under additional restrictions on the coefficients, they also showed that (1.14) is necessary for exponential stability. Here, we complete the analysis of [17] proving that (1.14) is indeed necessary and sufficient for exponential stability. If $\chi_{\varsigma} \chi_{\tau} \neq 0$, we also demonstrate that $T(t)$ is (semiuniformly) polynomially stable with optimal decay rate $\sqrt{t}$.

III. Finally, we consider the Timoshenko-Gurtin-Pipkin (TGP) system

$$
\left\{\begin{array}{l}
\rho_{1} \varphi_{t t}-k\left(\varphi_{x}+\psi\right)_{x}=0, \\
\rho_{2} \psi_{t t}-b \psi_{x x}+k\left(\varphi_{x}+\psi\right)+\gamma \vartheta_{x}=0, \\
\rho_{3} \vartheta_{t}-\varpi \int_{0}^{\infty} g(s) \vartheta_{x x}(t-s) \mathrm{d} s+\gamma \psi_{x t}=0,
\end{array}\right.
$$

with the Dirichlet-Neumann-Dirichlet boundary conditions

$$
\varphi(0, t)=\varphi(\ell, t)=\psi_{x}(0, t)=\psi_{x}(\ell, t)=\vartheta(0, t)=\vartheta(\ell, t)=0
$$

and the appropriate initial data. Such a model can be obtained from (1.9) in the limit case $l=0$ (cf. Subsection 1.1). The exponential stability of the solution semigroup $U(t)$ associated to the TGP system (1.15) in the Dafermos history framework has been analyzed in [19], where it is 
shown that $U(t)$ is exponentially stable if and only if $\chi_{g}=0$. Here, we prove that when $\chi_{g} \neq 0$ the semigroup $U(t)$ is (semiuniformly) polynomially stable with optimal decay rate $\sqrt{t}$.

Remark 1.4. As anticipated in the Abstract, it is interesting to observe that (1.12) is compatible with (1.2). Such a feature has been already pointed out in [17] regarding (1.14), which is compatible with (1.2) as well. As already noticed, this does not happen when the Fourier law is employed, since condition (1.5) is not compatible with (1.2).

Remark 1.5. We shall not discuss the systems of second-order thermoelasticity with MaxwellCattaneo or Gurtin-Pipkin laws, since they have been already studied in the literature and their stability properties are well-understood. In particular, exponential stability is the general rule here, independently of the values of the structural constants (see e.g. [39, 43]).

Plan of the paper. In the next Section 2, we make some comments on the results described so far, and we compare them with some previous achievements on related models. We also mention few possible extensions of our analysis. The short Section 3 is devoted to the notation. In the subsequent Sections 4-6 we state rigorously our main results, whose proofs are carried out in the remaining Sections 7-13.

\section{COMMENTS, COMPARISONS AND EXTENSIONS}

I. The BMC system can be seen as a "particular case" of the BGP one. Indeed, taking in (1.9) the exponential kernels

$$
g_{\varsigma}(s)=\frac{1}{\varpi \varsigma} \mathrm{e}^{-\frac{s}{\varpi \varsigma}} \quad \text { and } \quad h_{\tau}(s)=\frac{1}{\varpi \tau} \mathrm{e}^{-\frac{s}{\varpi \tau}},
$$

it is readily seen that the heat-flux variables

$$
\begin{aligned}
& p(t)=-\varpi \int_{0}^{\infty} g_{\varsigma}(s) \vartheta_{x}(t-s) \mathrm{d} s \\
& q(t)=-\varpi \int_{0}^{\infty} h_{\tau}(s) \xi_{x}(t-s) \mathrm{d} s,
\end{aligned}
$$

satisfy the $5^{\text {th }}$ and the $7^{\text {th }}$ equation of (1.13). Moreover, since $\chi_{\varsigma}=\chi_{g_{\varsigma}}$ and $\chi_{\tau}=\chi_{h_{\tau}}$, the exponential stability condition (1.14) is formally recovered from (1.12). Actually, using the techniques of [19, Section 8], one can prove rigorously that the semigroup $S(t)$ corresponding to the choice (2.1) is exponentially stable if and only if the same does the semigroup $T(t)$. Adapting the same techniques, one can also prove that if $S(t)$ with the choice (2.1) is polynomially stable with decay rate $\sqrt{t}$ then the same does $T(t)$. In addition, the calculations in the proof of the optimality of the decay rate of $S(t)$ can be easily adapted to show the optimality of the decay rate of $T(t)$. We refer to Section 12 of the present paper for details.

The same philosophy can be pursued in the study of the stability properties of the TimoshenkoMaxwell-Cattaneo (TMC) system

$$
\left\{\begin{array}{l}
\rho_{1} \varphi_{t t}-k\left(\varphi_{x}+\psi\right)_{x}=0, \\
\rho_{2} \psi_{t t}-b \psi_{x x}+k\left(\varphi_{x}+\psi\right)+\gamma \vartheta_{x}=0, \\
\rho_{3} \vartheta_{t}+p_{x}+\gamma \psi_{x t}=0, \\
\varsigma \varpi p_{t}+p+\varpi \vartheta_{x}=0 .
\end{array}\right.
$$


As shown in $[15,46]$, the solution semigroup associated to the TMC system is exponentially stable if and only if $\chi_{\varsigma}=0$, and polynomially stable with optimal decay rate $\sqrt{t}$ when $\chi_{\varsigma} \neq 0$. In [19] it is proved that the semigroup $U(t)$ associated to the TGP system with the choice $g=g_{\varsigma}$ is exponentially stable if and only if the same does the semigroup associated to the TMC system. Moreover, arguing as in Section 12 of the present paper, one can prove that if $U(t)$ with the choice $g=g_{\varsigma}$ is polynomially stable with optimal decay rate $\sqrt{t}$ then the same does the semigroup associated to the TMC system. Hence, both the exponential and the polynomial stability results obtained in $[15,46]$ are recovered. We refrain from providing detailed proofs here, leaving them to the interested reader.

II. A key feature of the models considered in this work is that the only source of dissipation is given by the thermal effects. It goes without saying that other thermal damping are possible. For instance, one can neglect the temperature $\xi$ and consider the "reduced BGP system"

$$
\left\{\begin{array}{l}
\rho_{1} \varphi_{t t}-k\left(\varphi_{x}+\psi+l w\right)_{x}-l k_{0}\left(w_{x}-l \varphi\right)=0, \\
\rho_{2} \psi_{t t}-b \psi_{x x}+k\left(\varphi_{x}+\psi+l w\right)+\gamma \vartheta_{x}=0, \\
\rho_{1} w_{t t}-k_{0}\left(w_{x}-l \varphi\right)_{x}+l k\left(\varphi_{x}+\psi+l w\right)=0, \\
\rho_{3} \vartheta_{t}-\varpi \int_{0}^{\infty} g(s) \vartheta_{x x}(t-s) \mathrm{d} s+\gamma \psi_{x t}=0 .
\end{array}\right.
$$

This model has been analyzed in [14], where it is shown that exponential stability occurs if and only if

$$
\chi_{g}=0 \quad \text { and } \quad \chi_{1}=0 .
$$

On the other hand, one can neglect the temperature $\vartheta$ and consider the reduced system

$$
\left\{\begin{array}{l}
\rho_{1} \varphi_{t t}-k\left(\varphi_{x}+\psi+l w\right)_{x}-l k_{0}\left(w_{x}-l \varphi\right)+l \gamma \xi=0, \\
\rho_{2} \psi_{t t}-b \psi_{x x}+k\left(\varphi_{x}+\psi+l w\right)=0, \\
\rho_{1} w_{t t}-k_{0}\left(w_{x}-l \varphi\right)_{x}+l k\left(\varphi_{x}+\psi+l w\right)+\gamma \xi_{x}=0, \\
\rho_{3} \xi_{t}-\varpi \int_{0}^{\infty} h(s) \xi_{x x}(t-s) \mathrm{d} s+\gamma\left(w_{x t}-l \varphi_{t}\right)=0 .
\end{array}\right.
$$

To the best of our knowledge, the latter has not been studied so far in the literature, but we conjecture that the necessary and sufficient condition for its exponential stability is

$$
\chi_{h}=0 \quad \text { and } \quad \chi_{0}=0 .
$$

In the light of the previous discussions, this insight becomes clear if one considers the MaxwellCattaneo version of (2.4)

$$
\left\{\begin{array}{l}
\rho_{1} \varphi_{t t}-k\left(\varphi_{x}+\psi+l w\right)_{x}-l k_{0}\left(w_{x}-l \varphi\right)+l \gamma \xi=0, \\
\rho_{2} \psi_{t t}-b \psi_{x x}+k\left(\varphi_{x}+\psi+l w\right)=0, \\
\rho_{1} w_{t t}-k_{0}\left(w_{x}-l \varphi\right)_{x}+l k\left(\varphi_{x}+\psi+l w\right)+\gamma \xi_{x}=0, \\
\rho_{3} \xi_{t}+q_{x}+\gamma\left(w_{x t}-l \varphi_{t}\right)=0, \\
\tau \varpi q_{t}+q+\varpi \xi_{x}=0,
\end{array}\right.
$$


where, as shown in [16], exponential stability occurs if and only if

$$
\chi_{\tau}=0 \quad \text { and } \quad \chi_{0}=0 .
$$

Note that both conditions (2.3) and (2.5) are incompatible with (1.2).

One might think that the exponential stability analysis of (2.2) and (2.4) is more challenging with respect to the one of (1.9), since the latter has a stronger damping mechanism contributed by two equations. Such a thought appears unfounded. Indeed, as for the BF system, in (1.9) the variables $\psi$ and $w$ are effectively damped but the variable $\varphi$ is only indirectly damped (cf. the discussion at the end of Subsection 1.2). In order to achieve the exponential stability, one has to "enucleate" the damping contribution of $\vartheta$ using the equality $\chi_{g}=0$ to stabilize exponentially the variable $\varphi$, and do the same for the damping contribution of $\xi$ using the equality $\chi_{h}=0$. As in the BF system, these conditions provide appropriate cancellations of some higher-order terms that pop up in the estimates. The exponential stability of (2.2) requires basically half of the job: in (2.2) only the variable $\psi$ is effectively damped, so that one has to use the equality $\chi_{g}=0$ to stabilize exponentially the variable $\varphi$ and the equality $\chi_{1}=0$ to stabilize exponentially the variable $w$, but the condition $\chi_{1}=0$ is quite easy to exploit (see [14, Section 6]). Similar remarks apply in the analysis of (2.4).

III. The methodology in this paper is based on resolvent estimates combined with the abstract results of L. Gearhart \& J. Prüss, A. Borichev \& Y. Tomilov and C.J.K. Batty \& T. Duyckaerts $[3,4,24,42]$. Although this approach is not new, the analysis of the present work presents some peculiar elements. We highlight the following aspects.

- In order to show the optimality of the polynomial decay rate, one needs to exploit sharp lower resolvent estimates which require the use of a quantified version of the RiemannLebesgue lemma recently obtained in [18].

- We provide a theoretical method to show that the polynomial decay rate $\sqrt{t}$ of the BGP system with the choice (2.1) implies automatically the same polynomial decay rate of the BMC system. It is very likely that such a method can be successfully applied to other models too.

- The study of the BGP and the TGP systems presents some difficulties connected with a structural lack of compactness which complicates the spectral analysis of the infinitesimal generators (see Remark 7.11 for details).

- The complexity of the BGP system (three wave equations coupled with two integrodifferential hyperbolic equations) requires a rather heavy technical effort when performing the resolvent estimates.

IV. In the limit case $\varsigma=\tau=0$, the BMC system (formally) reduces to the BF system, and condition (1.14) boils down to (1.5). Actually, as noticed in [14, 19], the BF system can be recovered directly from the BGP system by means of an appropriate singular limit procedure in which the kernels approach the Dirac mass at zero $\delta_{0}$. More precisely, for $\varepsilon>0$, let us set

$$
g_{\varepsilon}(s)=\frac{1}{\varepsilon} g\left(\frac{s}{\varepsilon}\right) \quad \text { and } \quad h_{\varepsilon}(s)=\frac{1}{\varepsilon} h\left(\frac{s}{\varepsilon}\right) .
$$


Since $g_{\varepsilon} \rightarrow \delta_{0}$ and $h_{\varepsilon} \rightarrow \delta_{0}$ in the distributional sense as $\varepsilon \rightarrow 0$, system (1.9) with the choice $g=g_{\varepsilon}$ and $h=h_{\varepsilon}$ (formally) boils down to (1.1) in the limit $\varepsilon \rightarrow 0$. Note also that

$$
\chi_{g_{\varepsilon}} \rightarrow-\frac{\rho_{1}}{k} \chi_{0} \quad \text { and } \quad \chi_{h_{\varepsilon}} \rightarrow-\frac{\rho_{1}}{k} \chi_{1}
$$

when $\varepsilon \rightarrow 0$, so that condition (1.12) reduces to (1.5). The same phenomenon appears in the passage from the TGP system (1.15) to the TF system (1.3) (see [19] for details). A rigorous proof of the convergence of solutions to equations with memory through the ones of the limit equation when the kernel collapses into a Dirac mass has been given in [10].

V. As pointed out in $[14,19]$, once the exponential stability properties of the BGP system are known it is possible to characterize the ones of the Bresse-Coleman-Gurtin system

$$
\left\{\begin{array}{l}
\rho_{1} \varphi_{t t}-k\left(\varphi_{x}+\psi+l w\right)_{x}-l k_{0}\left(w_{x}-l \varphi\right)+l \gamma \xi=0, \\
\rho_{2} \psi_{t t}-b \psi_{x x}+k\left(\varphi_{x}+\psi+l w\right)+\gamma \vartheta_{x}=0, \\
\rho_{1} w_{t t}-k_{0}\left(w_{x}-l \varphi\right)_{x}+l k\left(\varphi_{x}+\psi+l w\right)+\gamma \xi_{x}=0, \\
\rho_{3} \vartheta_{t}-\varpi(1-m) \vartheta_{x x}-\varpi m \int_{0}^{\infty} g(s) \vartheta_{x x}(t-s) \mathrm{d} s+\gamma \psi_{x t}=0, \\
\rho_{3} \xi_{t}-\varpi(1-m) \xi_{x x}-\varpi m \int_{0}^{\infty} h(s) \xi_{x x}(t-s) \mathrm{d} s+\gamma\left(w_{x t}-l \varphi_{t}\right)=0 .
\end{array}\right.
$$

In the model above, $m \in(0,1)$ is a fixed parameter and the temperatures obey the parabolichyperbolic law introduced by B.D. Coleman and M.E. Gurtin in [9]. The limit cases $m=0$ and $m=1$ correspond to the BF system (1.1) and the BGP system (1.9), respectively. The solution semigroup associated to (2.7) in the Dafermos history framework is exponentially stable if and only if $\chi_{0} \chi_{1}=0$, meaning that the parabolic character prevails on the hyperbolic one. To see that, following the procedure introduced in [19], let us set for $\varepsilon>0$

$$
g_{\varepsilon}(s)=\frac{1-m}{\varepsilon} g\left(\frac{s}{\varepsilon}\right)+m g(s) \quad \text { and } \quad h_{\varepsilon}(s)=\frac{1-m}{\varepsilon} h\left(\frac{s}{\varepsilon}\right)+m h(s) .
$$

Since $g_{\varepsilon} \rightarrow(1-m) \delta_{0}+m g$ and $h_{\varepsilon} \rightarrow(1-m) \delta_{0}+m h$ in the distributional sense when $\varepsilon \rightarrow 0$, system (1.9) with the choice $g=g_{\varepsilon}$ and $h=h_{\varepsilon}$ (formally) boils down to (2.7) as $\varepsilon \rightarrow 0$. At the same time, we have the convergence

$$
\chi_{g_{\varepsilon}} \rightarrow-\frac{\rho_{1}}{k} \chi_{0} \quad \text { and } \quad \chi_{h_{\varepsilon}} \rightarrow-\frac{\rho_{1}}{k} \chi_{1}
$$

for $\varepsilon \rightarrow 0$, so that condition (1.12) reduces to $\chi_{0} \chi_{1}=0$.

VI. As mentioned in the last section of [17], it is possible to consider "mixed Bresse models" where the temperatures obey two different thermal laws. For instance, one can study a system in which $\vartheta$ satisfies the Gurtin-Pipkin law and $\xi$ the Maxwell-Cattaneo one (and vice versa), or in which $\vartheta$ satisfies the Gurtin-Pipkin law and $\xi$ the Fourier one (and vice versa), and other combinations (including the Coleman-Gurtin law). All the exponential stability conditions for these systems can be derived from (1.12) by choosing appropriate kernels as in (2.1) or through appropriate singular limit procedures as above. For instance, if $\vartheta$ satisfies the Gurtin-Pipkin law and $\xi$ the Maxwell-Cattaneo one the exponential stability condition reads $\chi_{g} \chi_{\tau}=0$, while if $\vartheta$ satisfies the Gurtin-Pipkin law and $\xi$ the Fourier one it reads $\chi_{g} \chi_{1}=0$, and so on. 


\section{NOTATION}

The notation is mostly standard throughout. In particular, $\mathbb{R}^{+} \doteq(0, \infty)$ denotes the positive half-line, $\mathbb{N}=1,2,3, \ldots$ the set of positive integers, and $i \mathbb{R}$ the imaginary axis in the complex plane. The "Big O" and "Little-o" notations for functions or sequences have the standard meaning. Given a closed linear operator $L$ acting on a complex Hilbert space, we denote the domain by $\mathfrak{D}(\mathrm{L})$, the resolvent set by $\varrho(\mathrm{L})$ and the spectrum by $\sigma(\mathrm{L})$. The symbols $L^{2}, H^{1}, H_{0}^{1}, H^{2}$ indicate the usual complex Lebesgue and Sobolev spaces on the interval $(0, \ell)$, while $\langle\cdot, \cdot\rangle$ and $\|\cdot\|$ stand for the standard inner product and norm on $L^{2}$. Since no confusion can occur, the symbol $\|\cdot\|$ will be also used to denote the operator norm. We will also work with the Hilbert spaces of zero-mean functions

$$
L_{*}^{2}=\left\{f \in L^{2}: \int_{0}^{\ell} f(x) \mathrm{d} x=0\right\} \quad \text { and } \quad H_{*}^{1}=H^{1} \cap L_{*}^{2},
$$

the latter equipped with the gradient norm (due to the Poincare inequality). Along the paper, we routinely employ the Young, Hölder and Poincaré inequalities without explicit mention.

\section{Rigorous Statements FOR THE BGP System}

4.1. Assumptions on the kernels. The convolution kernels $g$ and $h$ are nonnegative bounded convex summable functions, both of unitary total mass and having the explicit form

$$
g(s)=\int_{s}^{\infty} \mu(r) \mathrm{d} r \quad \text { and } \quad h(s)=\int_{s}^{\infty} \nu(r) \mathrm{d} r
$$

where $\mu, \nu: \mathbb{R}^{+} \rightarrow \mathbb{R}^{+}$, called memory kernels, are nonincreasing absolutely continuous functions. In particular, $\mu$ and $\nu$ are summable with

$$
\int_{0}^{\infty} \mu(r) \mathrm{d} r=g(0) \quad \text { and } \quad \int_{0}^{\infty} \nu(r) \mathrm{d} r=h(0) .
$$

We also require that $\mu$ and $\nu$ are bounded about zero, namely

$$
\mu(0) \doteq \lim _{s \rightarrow 0} \mu(s)<\infty \quad \text { and } \quad \nu(0) \doteq \lim _{s \rightarrow 0} \nu(s)<\infty .
$$

Finally, we assume the so-called Dafermos conditions

$$
\begin{aligned}
& \mu^{\prime}(s)+\delta_{\mu} \mu(s) \leq 0, \\
& \nu^{\prime}(s)+\delta_{\nu} \nu(s) \leq 0,
\end{aligned}
$$

for some $\delta_{\mu}, \delta_{\nu}>0$ and almost every $s>0$.

4.2. Memory spaces. We introduce the so-called memory spaces

$$
\mathcal{M}=L_{\mu}^{2}\left(\mathbb{R}^{+} ; H_{0}^{1}\right) \quad \text { and } \quad \mathcal{N}=L_{\nu}^{2}\left(\mathbb{R}^{+} ; H_{0}^{1}\right)
$$

of square summable $H_{0}^{1}$-valued functions on $\mathbb{R}^{+}$with respect to the measures $\mu(s) \mathrm{d} s$ and $\nu(s) \mathrm{d} s$, respectively, endowed with the inner products

$$
\begin{aligned}
\left\langle\eta_{1}, \eta_{2}\right\rangle_{\mathcal{M}} & =\int_{0}^{\infty} \mu(s)\left\langle\eta_{1 x}(s), \eta_{2 x}(s)\right\rangle \mathrm{d} s \\
\left\langle\xi_{1}, \xi_{2}\right\rangle_{\mathcal{N}} & =\int_{0}^{\infty} \nu(s)\left\langle\xi_{1 x}(s), \xi_{2 x}(s)\right\rangle \mathrm{d} s .
\end{aligned}
$$


The induced norms will be denoted by $\|\cdot\|_{\mathcal{M}}$ and $\|\cdot\|_{\mathcal{N}}$. Moreover, we consider the infinitesimal generator of the right-translation semigroup on $\mathcal{M}$, that is, the operator

$$
T \eta=-\eta^{\prime} \quad \text { with } \quad \mathfrak{D}(T)=\left\{\eta \in \mathcal{M}: \eta^{\prime} \in \mathcal{M}, \lim _{s \rightarrow 0}\left\|\eta_{x}(s)\right\|=0\right\},
$$

where $\eta^{\prime}$ stands for the weak derivative with respect to the variable $s \in \mathbb{R}^{+}$. We will also work with the infinitesimal generator of the right-translation semigroup on $\mathcal{N}$, denoted with the same symbol $T$ and defined in the same way.

4.3. Extended memory space. We introduce the so-called extended memory space

$$
\mathcal{H}=H_{0}^{1} \times L^{2} \times H_{*}^{1} \times L_{*}^{2} \times H_{*}^{1} \times L_{*}^{2} \times L^{2} \times \mathcal{M} \times L^{2} \times \mathcal{N}
$$

equipped with the norm

$$
\begin{aligned}
\|u\|_{\mathcal{H}}^{2}= & k\left\|\varphi_{x}+\psi+l w\right\|^{2}+\rho_{1}\|\Phi\|^{2}+b\left\|\psi_{x}\right\|^{2}+\rho_{2}\|\Psi\|^{2}+k_{0}\left\|w_{x}-l \varphi\right\|^{2} \\
& +\rho_{1}\|W\|^{2}+\rho_{3}\|\vartheta\|^{2}+\varpi\|\eta\|_{\mathcal{M}}^{2}+\rho_{3}\|\xi\|^{2}+\varpi\|\zeta\|_{\mathcal{N}}^{2}
\end{aligned}
$$

for every $u=(\varphi, \Phi, \psi, \Psi, w, W, \vartheta, \eta, \xi, \zeta) \in \mathcal{H}$. The inner product associated to $\|\cdot\|_{\mathcal{H}}$ will be denoted by $\langle\cdot, \cdot\rangle_{\mathcal{H}}$. As customary in the analysis of Bresse systems, we are tacitly assuming that

$$
l \ell \neq n \pi, \quad \forall n \in \mathbb{N} .
$$

In fact, if (4.3) is violated it is not difficult to construct nonzero $u \in \mathcal{H}$ with $\|u\|_{\mathcal{H}}=0$. Instead, when (4.3) holds true, $\|\cdot\|_{\mathcal{H}}$ becomes a norm on $\mathcal{H}$, equivalent to the standard product norm

$$
|u|_{\mathcal{H}}^{2}=\left\|\varphi_{x}\right\|^{2}+\|\Phi\|^{2}+\left\|\psi_{x}\right\|^{2}+\|\Psi\|^{2}+\left\|w_{x}\right\|^{2}+\|W\|^{2}+\|\vartheta\|^{2}+\|\eta\|_{\mathcal{M}}^{2}+\|\xi\|^{2}+\|\zeta\|_{\mathcal{N}}^{2} .
$$

In particular, there exists a structural constant $\mathfrak{c}>0$ such that

$$
\mathfrak{c}|u|_{\mathcal{H}} \leq\|u\|_{\mathcal{H}} \leq \frac{1}{\mathfrak{c}}|u|_{\mathcal{H}}, \quad \forall u \in \mathcal{H}
$$

Indeed, the second inequality above is immediate, and within (4.3) is not hard to check that $\|\cdot\|_{\mathcal{H}}$ is a Banach norm on $\mathcal{H}$. Thus (4.4) follows from the Open Mapping Theorem. Along the paper, relation (4.4) will be tacitly employed in several occasions.

4.4. The semigroup. We reformulate the BGP system (1.9) making use of the history framework of Dafermos [12]. To this end, for $s>0$, we consider the auxiliary variables

$$
\eta^{t}(x, s)=\int_{0}^{s} \vartheta(x, t-r) \mathrm{d} r \quad \text { and } \quad \zeta^{t}(x, s)=\int_{0}^{s} \xi(x, t-r) \mathrm{d} r
$$


and we rewrite (1.9) in the form

$$
\left\{\begin{array}{l}
\rho_{1} \varphi_{t t}-k\left(\varphi_{x}+\psi+l w\right)_{x}-l k_{0}\left(w_{x}-l \varphi\right)+l \gamma \xi=0, \\
\rho_{2} \psi_{t t}-b \psi_{x x}+k\left(\varphi_{x}+\psi+l w\right)+\gamma \vartheta_{x}=0 \\
\rho_{1} w_{t t}-k_{0}\left(w_{x}-l \varphi\right)_{x}+l k\left(\varphi_{x}+\psi+l w\right)+\gamma \xi_{x}=0 \\
\rho_{3} \vartheta_{t}-\varpi \int_{0}^{\infty} \mu(s) \eta_{x x}(s) \mathrm{d} s+\gamma \psi_{x t}=0 \\
\eta_{t}=T \eta+\vartheta \\
\rho_{3} \xi_{t}-\varpi \int_{0}^{\infty} \nu(s) \zeta_{x x}(s) \mathrm{d} s+\gamma\left(w_{x t}-l \varphi_{t}\right)=0 \\
\zeta_{t}=T \zeta+\xi
\end{array}\right.
$$

Introducing the state vector $u(t)=\left(\varphi(t), \Phi(t), \psi(t), \Psi(t), w(t), W(t), \vartheta(t), \eta^{t}, \xi(t), \zeta^{t}\right) \in \mathcal{H}$, we view (4.5) as the abstract ODE on $\mathcal{H}$

$$
\frac{\mathrm{d}}{\mathrm{d} t} u(t)=\mathrm{A} u(t)
$$

where the linear operator $A$ is defined as

$$
\mathrm{A}\left(\begin{array}{c}
\varphi \\
\Phi \\
\psi \\
\Psi \\
w \\
W \\
\vartheta \\
\eta \\
\xi \\
\zeta
\end{array}\right)=\left(\begin{array}{c}
\Phi \\
\frac{k}{\rho_{1}}\left(\varphi_{x}+\psi+l w\right)_{x}+\frac{l k_{0}}{\rho_{1}}\left(w_{x}-l \varphi\right)-\frac{l \gamma}{\rho_{1}} \xi \\
\Psi \\
\frac{b}{\rho_{2}} \psi_{x x}-\frac{k}{\rho_{2}}\left(\varphi_{x}+\psi+l w\right)-\frac{\gamma}{\rho_{2}} \vartheta_{x} \\
W \\
\frac{k_{0}}{\rho_{1}}\left(w_{x}-l \varphi\right)_{x}-\frac{l k}{\rho_{1}}\left(\varphi_{x}+\psi+l w\right)-\frac{\gamma}{\rho_{1}} \xi_{x} \\
\frac{\varpi}{\rho_{3}} \int_{0}^{\infty} \mu(s) \eta_{x x}(s) \mathrm{d} s-\frac{\gamma}{\rho_{3}} \Psi_{x} \\
T \eta+\vartheta \\
\frac{\varpi}{\rho_{3}} \int_{0}^{\infty} \nu(s) \zeta_{x x}(s) \mathrm{d} s-\frac{\gamma}{\rho_{3}}\left(W_{x}-l \Phi\right) \\
T \zeta+\xi
\end{array}\right)
$$

with domain

$$
\mathfrak{D}(\mathrm{A})=\left\{\begin{array}{l|c}
\varphi \in \mathcal{H} & \begin{array}{c}
\varphi \\
\Phi, H_{x}, w_{x} \in H_{0}^{1} \\
\Psi, W \in H^{1} \\
\vartheta, \xi \in H_{0}^{1} \\
\\
\int_{0}^{\infty} \mu(s) \eta(s) \mathrm{d} s, \int_{0}^{\infty} \nu(s) \zeta(s) \mathrm{d} s \in H^{2} \\
\eta, \zeta \in \mathfrak{D}(T)
\end{array}
\end{array}\right\} .
$$

For every $u \in \mathfrak{D}(\mathrm{A})$, a straightforward computation entails

$$
\mathfrak{R e}\langle\mathrm{A} u, u\rangle_{\mathcal{H}}=\varpi \mathfrak{R e}\langle T \eta, \eta\rangle_{\mathcal{M}}+\varpi \mathfrak{R e}\langle T \zeta, \zeta\rangle_{\mathcal{N}}
$$

Moreover, as shown in [25], we have the equality

$$
\mathfrak{R e}\langle T \eta, \eta\rangle_{\mathcal{M}}+\mathfrak{R e}\langle T \zeta, \zeta\rangle_{\mathcal{N}}=-\frac{1}{2}[\Gamma[\eta]+\Gamma[\zeta]],
$$


where we set

$$
\Gamma[\eta]=\int_{0}^{\infty}-\mu^{\prime}(s)\left\|\eta_{x}(s)\right\|^{2} \mathrm{~d} s \quad \text { and } \quad \Gamma[\zeta]=\int_{0}^{\infty}-\nu^{\prime}(s)\left\|\zeta_{x}(s)\right\|^{2} \mathrm{~d} s .
$$

Since $\Gamma[\eta]$ and $\Gamma[\zeta]$ are nonnegative, we are led to

$$
\mathfrak{R e}\langle\mathrm{A} u, u\rangle_{\mathcal{H}}=-\frac{\varpi}{2}[\Gamma[\eta]+\Gamma[\zeta]] \leq 0,
$$

meaning that $A$ is dissipative. In addition, by means of standard techniques based on the LaxMilgram theorem, one can prove that the operator $1-A$ is surjective (see e.g. $[11,13]$ for details on the procedure in the context of equations with memory). In particular, $A$ is densely defined [41, Theorem 4.6] and, due to the Lumer-Phillips theorem, it generates a contraction semigroup

$$
S(t)=\mathrm{e}^{t \mathrm{~A}}: \mathcal{H} \rightarrow \mathcal{H}
$$

In particular, for every initial datum $u_{0} \in \mathcal{H}$, equation (4.6) admits a unique (mild) solution $u$ given by $u(t)=S(t) u_{0}$. If $u_{0} \in \mathfrak{D}(\mathrm{A})$, then the solution $u$ is classical (see e.g. [41]).

4.5. The results. The rigorous statements of the stability results for the BGP system anticipated in the Introduction read as follows $\left(\chi_{g}\right.$ and $\chi_{h}$ have been defined in Subsection 1.4).

Theorem 4.1. Assume that $\chi_{g} \chi_{h}=0$. Then the semigroup $S(t)$ is exponentially stable, namely, there exist two structural constants $\omega>0$ and $K=K(\omega) \geq 1$ such that

$$
\|S(t)\| \leq K \mathrm{e}^{-\omega t}, \quad \forall t \geq 0 .
$$

Theorem 4.2. The semigroup $S(t)$ is polynomially semiuniformly stable with decay rate $\sqrt{t}$, namely, $0 \in \varrho(\mathrm{A})$ and there exists a structural constant $K>0$ such that

$$
\left\|S(t) \mathrm{A}^{-1}\right\| \leq \frac{K}{\sqrt{t}}, \quad \forall t>0 .
$$

If in addition $\chi_{g} \chi_{h} \neq 0$, then such a decay rate is optimal, namely

$$
\limsup _{t \rightarrow \infty} \sqrt{t}\left\|S(t) \mathrm{A}^{-1}\right\|>0 .
$$

It is readily seen that condition (4.9) can be reformulated as

$$
\left\|S(t) u_{0}\right\|_{\mathcal{H}} \leq \frac{K}{\sqrt{t}}\left\|\mathrm{~A} u_{0}\right\|_{\mathcal{H}}, \quad \forall t>0, \quad \forall u_{0} \in \mathfrak{D}(\mathrm{A}) .
$$

Thus, for all $u_{0} \in \mathfrak{D}(\mathrm{A})$, we have the convergence $S(t) u_{0} \rightarrow 0$ as $t \rightarrow \infty$. Since $S(t)$ is a contraction semigroup, we conclude that

Corollary 4.3. The semigroup $S(t)$ is stable, namely, for every fixed $u_{0} \in \mathcal{H}$ we have

$$
\lim _{t \rightarrow \infty}\left\|S(t) u_{0}\right\|_{\mathcal{H}}=0 .
$$

When $\chi_{g} \chi_{h} \neq 0$, relation (4.10) tells that $S(t)$ cannot be exponentially stable. Hence, we get Corollary 4.4. The semigroup $S(t)$ is exponentially stable if and only if $\chi_{g} \chi_{h}=0$. 


\section{Rigorous StATEMENTS FOR THE BMC System}

We begin by introducing the product space

$$
\mathcal{V}=H_{0}^{1} \times L^{2} \times H_{*}^{1} \times L_{*}^{2} \times H_{*}^{1} \times L_{*}^{2} \times L^{2} \times L_{*}^{2} \times L^{2} \times L_{*}^{2}
$$

equipped with the norm

$$
\begin{aligned}
\|v\|_{\mathcal{V}}^{2}= & k\left\|\varphi_{x}+\psi+l w\right\|^{2}+\rho_{1}\|\Phi\|^{2}+b\left\|\psi_{x}\right\|^{2}+\rho_{2}\|\Psi\|^{2}+k_{0}\left\|w_{x}-l \varphi\right\|^{2} \\
& +\rho_{1}\|W\|^{2}+\rho_{3}\|\vartheta\|^{2}+\varsigma\|p\|^{2}+\rho_{3}\|\xi\|^{2}+\tau\|q\|^{2}
\end{aligned}
$$

for every $v=(\varphi, \Phi, \psi, \Psi, w, W, \vartheta, p, \xi, q) \in \mathcal{V}$. As before, we tacitly assume that (4.3) is satisfied, so that $\|\cdot\|_{\mathcal{V}}$ is a norm on $\mathcal{V}$, equivalent to the standard product norm. Then, we view the BMC system (1.13) as the abstract ODE on $\mathcal{V}$

$$
\frac{\mathrm{d}}{\mathrm{d} t} v(t)=\mathrm{B} v(t)
$$

where the linear operator $B$ is defined as

$$
\mathrm{B}\left(\begin{array}{c}
\varphi \\
\Phi \\
\psi \\
\Psi \\
w \\
W \\
\vartheta \\
p \\
\xi \\
q
\end{array}\right)=\left(\begin{array}{c}
\Phi \\
\frac{k}{\rho_{1}}\left(\varphi_{x}+\psi+l w\right)_{x}+\frac{l k_{0}}{\rho_{1}}\left(w_{x}-l \varphi\right)-\frac{l \gamma}{\rho_{1}} \xi \\
\Psi \\
\frac{b}{\rho_{2}} \psi_{x x}-\frac{k}{\rho_{2}}\left(\varphi_{x}+\psi+l w\right)-\frac{\gamma}{\rho_{2}} \vartheta_{x} \\
W \\
\frac{k_{0}}{\rho_{1}}\left(w_{x}-l \varphi\right)_{x}-\frac{l k}{\rho_{1}}\left(\varphi_{x}+\psi+l w\right)-\frac{\gamma}{\rho_{1}} \xi_{x} \\
-\frac{1}{\rho_{3}} p_{x}-\frac{\gamma}{\rho_{3}} \Psi_{x} \\
-\frac{1}{\varsigma \varpi} p-\frac{1}{\varsigma} \vartheta_{x} \\
-\frac{1}{\rho_{3}} q_{x}-\frac{\gamma}{\rho_{3}}\left(W_{x}-l \Phi\right) \\
-\frac{1}{\tau \varpi} q-\frac{1}{\tau} \xi_{x}
\end{array}\right)
$$

with domain

$$
\mathfrak{D}(\mathrm{B})=\left\{\begin{array}{l|c}
v \in \mathcal{V} & \begin{array}{c}
\varphi \in H^{2} \\
\Phi, \psi_{x}, w_{x} \in H_{0}^{1} \\
\Psi, W \in H^{1} \\
\vartheta, \xi \in H_{0}^{1} \\
p, q \in H^{1}
\end{array}
\end{array}\right\} .
$$

According to [17, Theorem 2.2], the operator B generates a contraction semigroup

$$
T(t)=\mathrm{e}^{t \mathrm{~B}}: \mathcal{V} \rightarrow \mathcal{V}
$$

In the same paper, the following are also shown $\left(\chi_{\tau}\right.$ and $\chi_{\varsigma}$ have been defined in Subsection 1.4).

- The inclusion $i \mathbb{R} \subset \varrho(\mathrm{B})$ holds.

- The semigroup $T(t)$ is exponentially stable when $\chi_{\varsigma} \chi_{\tau}=0$.

- The semigroup $T(t)$ is not exponentially stable when $\chi_{\varsigma} \chi_{\tau} \neq 0$ and the coefficients fulfill additional constraints (see [17, pp. 3594-3595]).

As mentioned in the Introduction, our result completes such an analysis. 
Theorem 5.1. The semigroup $T(t)$ is polynomially semiuniformly stable with decay rate $\sqrt{t}$, namely, there exists a structural constant $K>0$ such that

$$
\left\|T(t) \mathrm{B}^{-1}\right\| \leq \frac{K}{\sqrt{t}}, \quad \forall t>0 .
$$

If in addition $\chi_{\varsigma} \chi_{\tau} \neq 0$, then such a decay rate is optimal, namely

$$
\limsup _{t \rightarrow \infty} \sqrt{t}\left\|T(t) \mathrm{B}^{-1}\right\|>0 .
$$

Similarly to the BGP system, we also have

Corollary 5.2. The semigroup $T(t)$ is exponentially stable (if and) only if $\chi_{\varsigma} \chi_{\tau}=0$.

\section{Rigorous STATEMENTS FOR THE TGP System}

First, we consider the product space

$$
\mathcal{Z}=H_{0}^{1} \times L^{2} \times H_{*}^{1} \times L_{*}^{2} \times L^{2} \times \mathcal{M}
$$

equipped with the norm (equivalent to the standard product norm)

$$
\|z\|_{\mathcal{Z}}^{2}=k\left\|\varphi_{x}+\psi\right\|^{2}+\rho_{1}\|\Phi\|^{2}+b\left\|\psi_{x}\right\|^{2}+\rho_{2}\|\Psi\|^{2}+\rho_{3}\|\vartheta\|^{2}+\varpi\|\eta\|_{\mathcal{M}}^{2}
$$

for every $z=(\varphi, \Phi, \psi, \Psi, \vartheta, \eta) \in \mathcal{Z}$. The memory space $\mathcal{M}$ and its norm $\|\cdot\|_{\mathcal{M}}$ have been defined in Subsection 4.2. The inner product associated to $\|\cdot\|_{\mathcal{Z}}$ will be denoted by $\langle\cdot, \cdot\rangle_{\mathcal{Z}}$. Next, as in the BGP system, we introduce the auxiliary variable

$$
\eta^{t}(x, s)=\int_{0}^{s} \vartheta(x, t-r) \mathrm{d} r, \quad s>0,
$$

and we rewrite the TGP system (1.15) in the form

$$
\left\{\begin{array}{l}
\rho_{1} \varphi_{t t}-k\left(\varphi_{x}+\psi\right)_{x}=0 \\
\rho_{2} \psi_{t t}-b \psi_{x x}+k\left(\varphi_{x}+\psi\right)+\gamma \vartheta_{x}=0 \\
\rho_{3} \vartheta_{t}-\varpi \int_{0}^{\infty} \mu(s) \eta_{x x}(s) \mathrm{d} s+\gamma \psi_{x t}=0 \\
\eta_{t}=T \eta+\vartheta
\end{array}\right.
$$

The memory kernel $\mu$ has been defined in Subsection 4.1 and fulfills the properties stated therein (in particular, $\mu$ is bounded about zero and complies with (4.1)), while the operator $T$ has been defined in Subsection 4.2. As customary, we view (6.1) as the abstract ODE on $\mathcal{Z}$

where the linear operator $C$ reads

$$
\frac{\mathrm{d}}{\mathrm{d} t} z(t)=\mathrm{C} z(t)
$$

$$
\mathrm{C}\left(\begin{array}{c}
\varphi \\
\Phi \\
\psi \\
\Psi \\
\vartheta \\
\eta
\end{array}\right)=\left(\begin{array}{c}
\Phi \\
\frac{k}{\rho_{1}}\left(\varphi_{x}+\psi\right)_{x} \\
\Psi \\
\frac{b}{\rho_{2}} \psi_{x x}-\frac{k}{\rho_{2}}\left(\varphi_{x}+\psi\right)-\frac{\gamma}{\rho_{2}} \vartheta_{x} \\
\frac{w}{\rho_{3}} \int_{0}^{\infty} \mu(s) \eta_{x x}(s) \mathrm{d} s-\frac{\gamma}{\rho_{3}} \Psi_{x} \\
T \eta+\vartheta
\end{array}\right)
$$


with domain

$$
\mathfrak{D}(\mathrm{C})=\left\{\begin{array}{l|c}
z \in \mathcal{Z} & \begin{array}{c}
\varphi \in H^{2} \\
\Phi, \psi_{x} \in H_{0}^{1} \\
\Psi \in H^{1} \\
\vartheta \in H_{0}^{1} \\
\int_{0}^{\infty} \mu(s) \eta(s) \mathrm{d} s \in H^{2} \\
\eta \in \mathfrak{D}(T)
\end{array}
\end{array}\right\}
$$

According to [19, Theorem 3], the operator $C$ generates a contraction semigroup

$$
U(t)=\mathrm{e}^{t \boldsymbol{C}}: \mathcal{Z} \rightarrow \mathcal{Z}
$$

As mentioned in the Introduction, such a semigroup is exponentially stable if and only if $\chi_{g}=0$. Our result reads as follows.

Theorem 6.1. The semigroup $U(t)$ is polynomially semiuniformly stable with decay rate $\sqrt{t}$, namely, $0 \in \varrho(\mathrm{C})$ and there exists a structural constant $K>0$ such that

$$
\left\|U(t) \mathrm{C}^{-1}\right\| \leq \frac{K}{\sqrt{t}}, \quad \forall t>0 .
$$

If in addition $\chi_{g} \neq 0$, then such a decay rate is optimal, namely

$$
\limsup _{t \rightarrow \infty} \sqrt{t}\left\|U(t) C^{-1}\right\|>0 \text {. }
$$

The remaining of the paper is devoted to the proofs of Theorems 4.1, 4.2, 5.1 and 6.1.

\section{UPPER RESOlVENT Estimates FOR THE BGP System}

In this section, we establish some upper resolvent estimates that will be the key ingredients in order to prove Theorem 4.1 and the first part of Theorem 4.2. To this end, for every fixed $\lambda \in \mathbb{R}$ and $\widehat{u}=(\hat{\varphi}, \hat{\Phi}, \hat{\psi}, \hat{\Psi}, \hat{w}, \hat{W}, \hat{\vartheta}, \hat{\eta}, \hat{\xi}, \hat{\zeta}) \in \mathcal{H}$, we consider the resolvent equation

$$
\mathrm{i} \lambda u-\mathrm{A} u=\widehat{u}
$$

where $u=(\varphi, \Phi, \psi, \Psi, w, W, \vartheta, \eta, \xi, \zeta) \in \mathfrak{D}(\mathrm{A})$. In the sequel, we denote by $c>0$ a generic constant depending only on the structural quantities of the problem (hence independent of $\lambda$ ), whose value might change even within the same line.

The first step is to estimate the memory variables $\eta$ and $\zeta$. Multiplying the resolvent equation by $u$ in $\mathcal{H}$, taking the real part and using (4.7), we find

$$
\frac{\varpi}{2}[\Gamma[\eta]+\Gamma[\zeta]]=\mathfrak{R e}\langle\mathrm{i} \lambda u-\mathrm{A} u, u\rangle_{\mathcal{H}}=\mathfrak{R e}\langle\widehat{u}, u\rangle_{\mathcal{H}}
$$

As a consequence, we get the control

$$
\varpi[\Gamma[\eta]+\Gamma[\zeta]] \leq 2\|u\|_{\mathcal{H}}\|\widehat{u}\|_{\mathcal{H}}
$$

Exploiting (4.1) and (4.2), the inequality above yields the bounds

$$
\begin{aligned}
& \varpi\|\eta\|_{\mathcal{M}}^{2} \leq c\|u\|_{\mathcal{H}}\|\widehat{u}\|_{\mathcal{H}}, \\
& \varpi\|\zeta\|_{\mathcal{N}}^{2} \leq c\|u\|_{\mathcal{H}}\|\widehat{u}\|_{\mathcal{H}} .
\end{aligned}
$$


The next step is to estimate the remaining variables of $u$. To this aim, we write the resolvent equation componentwise

$$
\begin{aligned}
& \mathrm{i} \lambda \varphi-\Phi=\hat{\varphi}, \\
& \mathrm{i} \lambda \rho_{1} \Phi-k\left(\varphi_{x}+\psi+l w\right)_{x}-l k_{0}\left(w_{x}-l \varphi\right)+l \gamma \xi=\rho_{1} \hat{\Phi}, \\
& \mathrm{i} \lambda \psi-\Psi=\hat{\psi}, \\
& \mathrm{i} \lambda \rho_{2} \Psi-b \psi_{x x}+k\left(\varphi_{x}+\psi+l w\right)+\gamma \vartheta_{x}=\rho_{2} \hat{\Psi}, \\
& \mathrm{i} \lambda w-W=\hat{w}, \\
& \mathrm{i} \lambda \rho_{1} W-k_{0}\left(w_{x}-l \varphi\right)_{x}+l k\left(\varphi_{x}+\psi+l w\right)+\gamma \xi_{x}=\rho_{1} \hat{W}, \\
& \mathrm{i} \lambda \rho_{3} \vartheta-\varpi \int_{0}^{\infty} \mu(s) \eta_{x x}(s) \mathrm{d} s+\gamma \Psi_{x}=\rho_{3} \hat{\vartheta}, \\
& \mathrm{i} \lambda \eta-T \eta-\vartheta=\hat{\eta}, \\
& \mathrm{i} \lambda \rho_{3} \xi-\varpi \int_{0}^{\infty} \nu(s) \zeta_{x x}(s) \mathrm{d} s+\gamma\left(W_{x}-l \Phi\right)=\rho_{3} \hat{\xi}, \\
& \mathrm{i} \lambda \zeta-T \zeta-\xi=\hat{\zeta},
\end{aligned}
$$

and we establish a number of auxiliary lemmas.

Lemma 7.1. For every $\varepsilon \in(0,1)$ the inequality

$$
\rho_{3}\|\vartheta\|^{2} \leq \varepsilon\|\Psi\|^{2}+\frac{c}{\varepsilon}\|u\|_{\mathcal{H}}\|\widehat{u}\|_{\mathcal{H}}
$$

holds for some structural constant $c>0$ independent of $\varepsilon$ and $\lambda$.

Proof. We introduce the space $\mathcal{M}_{0}=L_{\mu}^{2}\left(\mathbb{R}^{+} ; L^{2}\right)$ equipped with the inner product

$$
\left\langle\eta_{1}, \eta_{2}\right\rangle_{\mathcal{M}_{0}}=\int_{0}^{\infty} \mu(s)\left\langle\eta_{1}(s), \eta_{2}(s)\right\rangle \mathrm{d} s .
$$

Noting that $\mathcal{M} \subset \mathcal{M}_{0}$ with continuous inclusion, we multiply (7.11) by $\vartheta$ in $\mathcal{M}_{0}$ and we get

$$
g(0)\|\vartheta\|^{2}=\mathrm{i} \lambda\langle\eta, \vartheta\rangle_{\mathcal{M}_{0}}-\langle T \eta, \vartheta\rangle_{\mathcal{M}_{0}}-\langle\hat{\eta}, \vartheta\rangle_{\mathcal{M}_{0}}
$$

Exploiting (7.10), we rewrite

$$
\begin{aligned}
\mathrm{i} \lambda\langle\eta, \vartheta\rangle_{\mathcal{M}_{0}}= & \frac{\varpi}{\rho_{3}} \int_{0}^{\infty} \mu(s) \int_{0}^{\infty} \mu(r)\left\langle\eta_{x}(s), \eta_{x}(r)\right\rangle \mathrm{d} r \mathrm{~d} s-\frac{\gamma}{\rho_{3}} \int_{0}^{\infty} \mu(s)\left\langle\eta_{x}(s), \Psi\right\rangle \mathrm{d} s \\
& -\int_{0}^{\infty} \mu(s)\langle\eta(s), \hat{\vartheta}\rangle \mathrm{d} s .
\end{aligned}
$$

Owing to the equality above and (7.2), we obtain the control

$$
\left|\mathrm{i} \lambda\langle\eta, \vartheta\rangle_{\mathcal{M}_{0}}\right| \leq c\|\Psi\|\|\eta\|_{\mathcal{M}}+c\|u\|_{\mathcal{H}}\|\widehat{u}\|_{\mathcal{H}}
$$

Moreover, integrating by parts in $s$, we infer that

$$
\langle T \eta, \vartheta\rangle_{\mathcal{M}_{0}}=\int_{0}^{\infty} \mu^{\prime}(s)\langle\eta(s), \vartheta\rangle \mathrm{d} s,
$$


where the boundary terms vanish by standard arguments (see e.g. [25]). Hence, invoking (7.1), we find the bound

$$
\left|\langle T \eta, \vartheta\rangle_{\mathcal{M}_{0}}\right| \leq c\|\vartheta\| \sqrt{\Gamma[\eta]} \leq \frac{g(0)}{2}\|\vartheta\|^{2}+c\|u\|_{\mathcal{H}}\|\widehat{u}\|_{\mathcal{H}}
$$

Finally, it is apparent that

$$
\left|\langle\hat{\eta}, \vartheta\rangle_{\mathcal{M}_{0}}\right| \leq c\|u\|_{\mathcal{H}}\|\widehat{u}\|_{\mathcal{H}}
$$

Collecting the estimates obtained so far and invoking (7.2), we end up with

$$
\rho_{3}\|\vartheta\|^{2} \leq c\|\Psi\|\|\eta\|_{\mathcal{M}}+c\|u\|_{\mathcal{H}}\|\widehat{u}\|_{\mathcal{H}} \leq \varepsilon\|\Psi\|^{2}+\frac{c}{\varepsilon}\|u\|_{\mathcal{H}}\|\widehat{u}\|_{\mathcal{H}}
$$

for every $\varepsilon \in(0,1)$, as claimed.

Lemma 7.2. For every $\varepsilon \in(0,1)$ the inequality

$$
\rho_{3}\|\xi\|^{2} \leq \varepsilon\|u\|_{\mathcal{H}}^{2}+\frac{c}{\varepsilon}\|u\|_{\mathcal{H}}\|\widehat{u}\|_{\mathcal{H}}
$$

holds for some structural constant $c>0$ independent of $\varepsilon$ and $\lambda$.

Proof. The argument is similar to the previous one. Setting $\mathcal{N}_{0}=L_{\nu}^{2}\left(\mathbb{R}^{+} ; L^{2}\right)$ endowed with the inner product

$$
\left\langle\xi_{1}, \xi_{2}\right\rangle_{\mathcal{N}_{0}}=\int_{0}^{\infty} \nu(s)\left\langle\xi_{1}(s), \xi_{2}(s)\right\rangle \mathrm{d} s
$$

and noting that $\mathcal{N} \subset \mathcal{N}_{0}$ with continuous inclusion, we multiply (7.13) by $\xi$ in $\mathcal{N}_{0}$ obtaining

$$
h(0)\|\xi\|^{2}=\mathrm{i} \lambda\langle\zeta, \xi\rangle_{\mathcal{N}_{0}}-\langle T \zeta, \xi\rangle_{\mathcal{N}_{0}}-\langle\hat{\zeta}, \xi\rangle_{\mathcal{N}_{0}}
$$

Making use of (7.12), we rewrite

$$
\begin{aligned}
\mathrm{i} \lambda\langle\zeta, \xi\rangle_{\mathcal{N}_{0}}= & \frac{\varpi}{\rho_{3}} \int_{0}^{\infty} \nu(s) \int_{0}^{\infty} \nu(r)\left\langle\zeta_{x}(s), \zeta_{x}(r)\right\rangle \mathrm{d} r \mathrm{~d} s-\frac{\gamma}{\rho_{3}} \int_{0}^{\infty} \nu(s)\left\langle\zeta_{x}(s), W\right\rangle \mathrm{d} s \\
& -\frac{l \gamma}{\rho_{3}} \int_{0}^{\infty} \nu(s)\langle\zeta(s), \Phi\rangle \mathrm{d} s-\int_{0}^{\infty} \nu(s)\langle\zeta(s), \hat{\xi}\rangle \mathrm{d} s .
\end{aligned}
$$

Due to the equality above and (7.3), we find the estimate

$$
\left|\mathrm{i} \lambda\langle\zeta, \xi\rangle_{\mathcal{N}_{0}}\right| \leq c\|u\|_{\mathcal{H}}\|\zeta\|_{\mathcal{N}}+c\|u\|_{\mathcal{H}}\|\widehat{u}\|_{\mathcal{H}}
$$

Integrating by parts in $s$ and exploiting (7.1), we also have

$$
\left|\langle T \zeta, \xi\rangle_{\mathcal{N}_{0}}\right| \leq c\|\xi\| \sqrt{\Gamma[\zeta]} \leq \frac{h(0)}{2}\|\xi\|^{2}+c\|u\|_{\mathcal{H}}\|\widehat{u}\|_{\mathcal{H}}
$$

Finally, it is readily seen that

$$
\left|\langle\hat{\zeta}, \xi\rangle_{\mathcal{N}_{0}}\right| \leq c\|u\|_{\mathcal{H}}\|\widehat{u}\|_{\mathcal{H}}
$$

Collecting these estimates and using (7.3), we conclude that

$$
\rho_{3}\|\xi\|^{2} \leq c\|u\|_{\mathcal{H}}\|\zeta\|_{\mathcal{N}}+c\|u\|_{\mathcal{H}}\|\widehat{u}\|_{\mathcal{H}} \leq \varepsilon\|u\|_{\mathcal{H}}^{2}+\frac{c}{\varepsilon}\|u\|_{\mathcal{H}}\|\widehat{u}\|_{\mathcal{H}}
$$

for every $\varepsilon \in(0,1)$. The proof is finished. 
Lemma 7.3. For every $\varepsilon \in(0,1)$ and every $\lambda \neq 0$ the inequality

$$
b\left\|\psi_{x}\right\|^{2} \leq \frac{\varepsilon}{|\lambda|^{2}}\|u\|_{\mathcal{H}}^{2}+c \varepsilon\|\Psi\|^{2}+\frac{c}{\varepsilon}\left[\frac{1}{|\lambda|}+1\right]\|u\|_{\mathcal{H}}\|\widehat{u}\|_{\mathcal{H}}
$$

holds for some structural constant $c>0$ independent of $\varepsilon$ and $\lambda$.

Proof. We preliminary show

$$
\left\|\vartheta_{x}\right\| \leq c[1+|\lambda|] \sqrt{\|u\|_{\mathcal{H}}\|\widehat{u}\|_{\mathcal{H}}}+c\|\hat{u}\|_{\mathcal{H}} .
$$

To this end, multiplying (7.11) by $\vartheta$ in $\mathcal{M}$, we get

$$
g(0)\left\|\vartheta_{x}\right\|^{2}=\mathrm{i} \lambda\langle\eta, \vartheta\rangle_{\mathcal{M}}-\langle T \eta, \vartheta\rangle_{\mathcal{M}}-\langle\hat{\eta}, \vartheta\rangle_{\mathcal{M}} .
$$

In the light of (7.2), we estimate

$$
\left|\mathrm{i} \lambda\langle\eta, \vartheta\rangle_{\mathcal{M}}\right| \leq c|\lambda|\left\|\vartheta_{x}\right\| \sqrt{\|u\|_{\mathcal{H}}\|\widehat{u}\|_{\mathcal{H}}}
$$

while integrating by parts in $s$ and exploiting (7.1) we infer that

$$
\left|\langle T \eta, \vartheta\rangle_{\mathcal{M}}\right| \leq c\left\|\vartheta_{x}\right\| \sqrt{\Gamma[\eta]} \leq c\left\|\vartheta_{x}\right\| \sqrt{\|u\|_{\mathcal{H}}\|\widehat{u}\|_{\mathcal{H}}}
$$

Finally, noting that

$$
\left|\langle\hat{\eta}, \vartheta\rangle_{\mathcal{M}}\right| \leq c\left\|\vartheta_{x}\right\|\|\widehat{u}\|_{\mathcal{H}},
$$

we arrive at (7.14). Next, substituting (7.6) into (7.10), we find the identity

$$
\mathrm{i} \lambda \gamma \psi_{x}=\varpi \int_{0}^{\infty} \mu(s) \eta_{x x}(s) \mathrm{d} s-\mathrm{i} \lambda \rho_{3} \vartheta+\gamma \hat{\psi}_{x}+\rho_{3} \hat{\vartheta} .
$$

A multiplication by $\psi_{x}$ in $L^{2}$ entails

$$
\mathrm{i} \lambda \gamma\left\|\psi_{x}\right\|^{2}=-\varpi \int_{0}^{\infty} \mu(s)\left\langle\eta_{x}(s), \psi_{x x}\right\rangle \mathrm{d} s-\mathrm{i} \lambda \rho_{3}\left\langle\vartheta, \psi_{x}\right\rangle+\gamma\left\langle\hat{\psi}_{x}, \psi_{x}\right\rangle+\rho_{3}\left\langle\hat{\vartheta}, \psi_{x}\right\rangle .
$$

With the aid of (7.7), we rewrite the first term in the right-hand side as

$$
\begin{aligned}
\int_{0}^{\infty} \mu(s)\left\langle\eta_{x}(s), \psi_{x x}\right\rangle \mathrm{d} s= & \frac{\gamma}{b} \int_{0}^{\infty} \mu(s)\left\langle\eta_{x}(s), \vartheta_{x}\right\rangle \mathrm{d} s-\frac{\mathrm{i} \lambda \rho_{2}}{b} \int_{0}^{\infty} \mu(s)\left\langle\eta_{x}(s), \Psi\right\rangle \mathrm{d} s \\
& +\frac{k}{b} \int_{0}^{\infty} \mu(s)\left\langle\eta_{x}(s), \varphi_{x}+\psi+l w\right\rangle \mathrm{d} s-\frac{\rho_{2}}{b} \int_{0}^{\infty} \mu(s)\left\langle\eta_{x}(s), \hat{\Psi}\right\rangle \mathrm{d} s .
\end{aligned}
$$

Due to the equality above, together with (7.2) and (7.14), we derive the bound

$$
\begin{aligned}
\left|\int_{0}^{\infty} \mu(s)\left\langle\eta_{x}(s), \psi_{x x}\right\rangle \mathrm{d} s\right| & \leq c\left[\|u\|_{\mathcal{H}}+|\lambda|\|\Psi\|\right]\|\eta\|_{\mathcal{M}}+c\left\|\vartheta_{x}\right\|\|\eta\|_{\mathcal{M}}+c\|u\|_{\mathcal{H}}\|\widehat{u}\|_{\mathcal{H}} \\
& \leq c\left[\|u\|_{\mathcal{H}}+|\lambda|\|\Psi\|\right]\|\eta\|_{\mathcal{M}}+c[1+|\lambda|]\|u\|_{\mathcal{H}}\|\widehat{u}\|_{\mathcal{H}} .
\end{aligned}
$$

As a consequence, from (7.15) we infer that

$$
|\lambda|\left\|\psi_{x}\right\|^{2} \leq c\left[\|u\|_{\mathcal{H}}+|\lambda|\|\Psi\|\right]\|\eta\|_{\mathcal{M}}+c|\lambda|\|\vartheta\|\left\|\psi_{x}\right\|+c[1+|\lambda|]\|u\|_{\mathcal{H}}\|\widehat{u}\|_{\mathcal{H}} .
$$

Invoking (7.2) once more and using Lemma 7.1, we arrive at

$$
\begin{aligned}
2 b\left\|\psi_{x}\right\|^{2} & \leq \frac{c}{|\lambda|}\|u\|_{\mathcal{H}}\|\eta\|_{\mathcal{M}}+c\|\Psi\|\|\eta\|_{\mathcal{M}}+c\|\vartheta\|\left\|\psi_{x}\right\|+c\left[\frac{1}{|\lambda|}+1\right]\|u\|_{\mathcal{H}}\|\widehat{u}\|_{\mathcal{H}} \\
& \leq \frac{\varepsilon}{|\lambda|^{2}}\|u\|_{\mathcal{H}}^{2}+c \varepsilon\|\Psi\|^{2}+b\left\|\psi_{x}\right\|^{2}+\frac{c}{\varepsilon}\left[\frac{1}{|\lambda|}+1\right]\|u\|_{\mathcal{H}}\|\widehat{u}\|_{\mathcal{H}}
\end{aligned}
$$


for every $\varepsilon \in(0,1)$ and every $\lambda \neq 0$. The conclusion follows.

Lemma 7.4. For every $\varepsilon>0$ small enough and every $\lambda \neq 0$ the inequality

$$
\rho_{2}\|\Psi\|^{2} \leq \frac{c \varepsilon}{|\lambda|^{2}}\|u\|_{\mathcal{H}}^{2}+\frac{c}{\varepsilon^{3}}\left[\frac{1}{|\lambda|}+1\right]\|u\|_{\mathcal{H}}\|\widehat{u}\|_{\mathcal{H}}
$$

holds for some structural constant $c>0$ independent of $\varepsilon$ and $\lambda$.

Proof. Introducing the primitive

$$
P_{\Psi}(x)=\int_{0}^{x} \Psi(y) \mathrm{d} y \in H_{0}^{1}
$$

and multiplying (7.10) by $P_{\Psi}$ in $L^{2}$, we find

$$
\gamma\|\Psi\|^{2}=\mathrm{i} \lambda \rho_{3}\left\langle\vartheta, P_{\Psi}\right\rangle+\varpi\left\langle\eta, P_{\Psi}\right\rangle_{\mathcal{M}}-\rho_{3}\left\langle\hat{\vartheta}, P_{\Psi}\right\rangle .
$$

Moreover, an integration of (7.7) on $(0, x)$ entails

$$
\mathrm{i} \lambda P_{\Psi}(x)=\frac{b}{\rho_{2}} \psi_{x}(x)-\frac{k}{\rho_{2}} \varphi(x)-\frac{\gamma}{\rho_{2}} \vartheta(x)-\frac{k}{\rho_{2}} \int_{0}^{x}[\psi(y)+l w(y)] \mathrm{d} y+\int_{0}^{x} \hat{\Psi}(y) \mathrm{d} y .
$$

Using the identity above, together with (7.4) and (7.8), we estimate the first term in the righthand side of (7.16) as

$$
\begin{aligned}
\left|\mathrm{i} \lambda \rho_{3}\left\langle\vartheta, P_{\Psi}\right\rangle\right| & \leq c[\|\varphi\|+\|w\|]\|\vartheta\|+c\left\|\psi_{x}\right\|\|\vartheta\|+c\|\vartheta\|^{2}+c\|u\|_{\mathcal{H}}\|\widehat{u}\|_{\mathcal{H}} \\
& \leq \frac{c}{|\lambda|}[\|\Phi\|+\|W\|]\|\vartheta\|+c\left\|\psi_{x}\right\|\|\vartheta\|+c\|\vartheta\|^{2}+c\left[\frac{1}{|\lambda|}+1\right]\|u\|_{\mathcal{H}}\|\widehat{u}\|_{\mathcal{H}} \\
& \leq \frac{c}{|\lambda|}\|u\|_{\mathcal{H}}\|\vartheta\|+c\left\|\psi_{x}\right\|^{2}+c\|\vartheta\|^{2}+c\left[\frac{1}{|\lambda|}+1\right]\|u\|_{\mathcal{H}}\|\widehat{u}\|_{\mathcal{H}},
\end{aligned}
$$

for every $\lambda \neq 0$. In the light of (7.2), the remaining terms in the right-hand side of (7.16) can be controlled as

$$
\left|\varpi\left\langle\eta, P_{\Psi}\right\rangle_{\mathcal{M}}-\rho_{3}\left\langle\hat{\vartheta}, P_{\Psi}\right\rangle\right| \leq c\|\Psi\|\|\eta\|_{\mathcal{M}}+c\|u\|_{\mathcal{H}}\|\widehat{u}\|_{\mathcal{H}} \leq \frac{\gamma}{2}\|\Psi\|^{2}+c\|u\|_{\mathcal{H}}\|\widehat{u}\|_{\mathcal{H}} .
$$

Thus, invoking Lemmas 7.1 and 7.3, we get

$$
\begin{aligned}
2 \rho_{2}\|\Psi\|^{2} & \leq \frac{c}{|\lambda|}\|u\|_{\mathcal{H}}\|\vartheta\|+c\left\|\psi_{x}\right\|^{2}+c\|\vartheta\|^{2}+c\left[\frac{1}{|\lambda|}+1\right]\|u\|_{\mathcal{H}}\|\widehat{u}\|_{\mathcal{H}} \\
& \leq \frac{\varepsilon}{|\lambda|^{2}}\|u\|_{\mathcal{H}}^{2}+c\left\|\psi_{x}\right\|^{2}+\frac{c}{\varepsilon}\|\vartheta\|^{2}+c\left[\frac{1}{|\lambda|}+1\right]\|u\|_{\mathcal{H}}\|\widehat{u}\|_{\mathcal{H}} \\
& \leq \frac{c \varepsilon}{|\lambda|^{2}}\|u\|_{\mathcal{H}}^{2}+\rho_{2}\|\Psi\|^{2}+\frac{c}{\varepsilon^{3}}\left[\frac{1}{|\lambda|}+1\right]\|u\|_{\mathcal{H}}\|\widehat{u}\|_{\mathcal{H}}
\end{aligned}
$$

for every $\varepsilon>0$ small enough and every $\lambda \neq 0$. The thesis has been reached.

Lemma 7.5. For every $\varepsilon \in(0,1)$ and every $\lambda \neq 0$ the inequality

$$
k_{0}\left\|w_{x}-l \varphi\right\|^{2} \leq c \varepsilon\left[\frac{1}{|\lambda|^{2}}+1\right]\|u\|_{\mathcal{H}}^{2}+\frac{c}{\varepsilon}\left[\frac{1}{|\lambda|}+1\right]\|u\|_{\mathcal{H}}\|\widehat{u}\|_{\mathcal{H}}
$$

holds for some structural constant $c>0$ independent of $\varepsilon$ and $\lambda$. 
Proof. We preliminary show

$$
\left\|\xi_{x}\right\| \leq c[1+|\lambda|] \sqrt{\|u\|_{\mathcal{H}}\|\widehat{u}\|_{\mathcal{H}}}+c\|\hat{u}\|_{\mathcal{H}} .
$$

To this aim, multiplying (7.13) by $\xi$ in $\mathcal{N}$, we get

$$
h(0)\left\|\xi_{x}\right\|^{2}=\mathrm{i} \lambda\langle\zeta, \xi\rangle_{\mathcal{N}}-\langle T \zeta, \xi\rangle_{\mathcal{N}}-\langle\hat{\zeta}, \xi\rangle_{\mathcal{N}}
$$

Making use of (7.3), it is readily seen that

$$
\left|\mathrm{i} \lambda\langle\zeta, \xi\rangle_{\mathcal{N}}\right| \leq c|\lambda|\left\|\xi_{x}\right\| \sqrt{\|u\|_{\mathcal{H}}\|\widehat{u}\|_{\mathcal{H}}},
$$

while integrating by parts in $s$ and owing to (7.1) we have

$$
\left|\langle T \zeta, \xi\rangle_{\mathcal{N}}\right| \leq c\left\|\xi_{x}\right\| \sqrt{\Gamma[\zeta]} \leq c\left\|\xi_{x}\right\| \sqrt{\|u\|_{\mathcal{H}}\|\widehat{u}\|_{\mathcal{H}}}
$$

Finally, we estimate

$$
\left|\langle\hat{\zeta}, \xi\rangle_{\mathcal{N}}\right| \leq c\left\|\xi_{x}\right\|\|\widehat{u}\|_{\mathcal{H}}
$$

and (7.17) follows. Next, invoking (7.4) and (7.8), we infer that

$$
W_{x}-l \Phi=\mathrm{i} \lambda\left(w_{x}-l \varphi\right)-\hat{w}_{x}+l \hat{\varphi}
$$

and plugging such an equality into (7.12) we obtain

$$
\mathrm{i} \lambda \gamma\left(w_{x}-l \varphi\right)=\varpi \int_{0}^{\infty} \nu(s) \zeta_{x x}(s) \mathrm{d} s-\mathrm{i} \lambda \rho_{3} \xi+\gamma\left(\hat{w}_{x}-l \hat{\varphi}\right)+\rho_{3} \hat{\xi} .
$$

Multiplying the identity above by $w_{x}-l \varphi$ in $L^{2}$, we are led to

$$
\begin{aligned}
\mathrm{i} \lambda \gamma\left\|w_{x}-l \varphi\right\|^{2}= & -\varpi \int_{0}^{\infty} \nu(s)\left\langle\zeta_{x}(s),\left(w_{x}-l \varphi\right)_{x}\right\rangle \mathrm{d} s-\mathrm{i} \lambda \rho_{3}\left\langle\xi, w_{x}-l \varphi\right\rangle \\
& +\gamma\left\langle\hat{w}_{x}-l \hat{\varphi}, w_{x}-l \varphi\right\rangle+\rho_{3}\left\langle\hat{\xi}, w_{x}-l \varphi\right\rangle .
\end{aligned}
$$

Exploiting (7.9), we rewrite the first term in the right-hand side as

$$
\begin{aligned}
\int_{0}^{\infty} \nu(s)\left\langle\zeta_{x}(s),\left(w_{x}-l \varphi\right)_{x}\right\rangle \mathrm{d} s= & \frac{\gamma}{k_{0}} \int_{0}^{\infty} \nu(s)\left\langle\zeta_{x}(s), \xi_{x}\right\rangle \mathrm{d} s-\frac{\mathrm{i} \lambda \rho_{1}}{k_{0}} \int_{0}^{\infty} \nu(s)\left\langle\zeta_{x}(s), W\right\rangle \mathrm{d} s \\
& +\frac{l k}{k_{0}} \int_{0}^{\infty} \nu(s)\left\langle\zeta_{x}(s), \varphi_{x}+\psi+l w\right\rangle \mathrm{d} s \\
& -\frac{\rho_{1}}{k_{0}} \int_{0}^{\infty} \nu(s)\left\langle\zeta_{x}(s), \hat{W}\right\rangle \mathrm{d} s .
\end{aligned}
$$

Making use of the equality above, together with (7.3) and (7.17), we derive the control

$$
\begin{aligned}
\left|\int_{0}^{\infty} \nu(s)\left\langle\zeta_{x}(s),\left(w_{x}-l \varphi\right)_{x}\right\rangle \mathrm{d} s\right| & \leq c[1+|\lambda|]\|u\|_{\mathcal{H}}\|\zeta\|_{\mathcal{N}}+c\left\|\xi_{x}\right\|\|\zeta\|_{\mathcal{N}}+c\|u\|_{\mathcal{H}}\|\widehat{u}\|_{\mathcal{H}} \\
& \leq c[1+|\lambda|]\|u\|_{\mathcal{H}}\|\zeta\|_{\mathcal{N}}+c[1+|\lambda|]\|u\|_{\mathcal{H}}\|\widehat{u}\|_{\mathcal{H}}
\end{aligned}
$$

As a consequence, from (7.18) we find

$$
|\lambda|\left\|w_{x}-l \varphi\right\|^{2} \leq c[1+|\lambda|]\|u\|_{\mathcal{H}}\|\zeta\|_{\mathcal{N}}+c|\lambda|\|\xi\|\left\|w_{x}-l \varphi\right\|+c[1+|\lambda|]\|u\|_{\mathcal{H}}\|\widehat{u}\|_{\mathcal{H}} .
$$

Appealing again to (7.3) and using Lemma 7.2, we finally get

$$
2 k_{0}\left\|w_{x}-l \varphi\right\|^{2} \leq c\left[\frac{1}{|\lambda|}+1\right]\|u\|_{\mathcal{H}}\|\zeta\|_{\mathcal{N}}+c\|\xi\|\left\|w_{x}-l \varphi\right\|+c\left[\frac{1}{|\lambda|}+1\right]\|u\|_{\mathcal{H}}\|\widehat{u}\|_{\mathcal{H}}
$$




$$
\leq c \varepsilon\left[\frac{1}{|\lambda|^{2}}+1\right]\|u\|_{\mathcal{H}}^{2}+k_{0}\left\|w_{x}-l \varphi\right\|^{2}+\frac{c}{\varepsilon}\left[\frac{1}{|\lambda|}+1\right]\|u\|_{\mathcal{H}}\|\widehat{u}\|_{\mathcal{H}}
$$

for every $\varepsilon \in(0,1)$ and every $\lambda \neq 0$. The lemma has been proved.

Lemma 7.6. For every $\varepsilon \in(0,1)$ and every $\lambda \neq 0$ the inequality

$$
\rho_{1}\|W\|^{2} \leq c \varepsilon\left[\frac{1}{|\lambda|^{2}}+1\right]\|u\|_{\mathcal{H}}^{2}+\frac{c}{\varepsilon|\lambda|^{2}}\left\|\varphi_{x}+\psi+l w\right\|^{2}+\frac{c}{\varepsilon^{3}}\left[\frac{1}{|\lambda|}+1\right]\|u\|_{\mathcal{H}}\|\widehat{u}\|_{\mathcal{H}}
$$

holds for some structural constant $c>0$ independent of $\varepsilon$ and $\lambda$.

Proof. Multiplying (7.9) by $w$ in $L^{2}$ and invoking (7.8), we have

$$
\rho_{1}\|W\|^{2}=k_{0}\left\langle w_{x}-l \varphi, w_{x}\right\rangle+l k\left\langle\varphi_{x}+\psi+l w, w\right\rangle-\gamma\left\langle\xi, w_{x}\right\rangle-\rho_{1}\langle W, \hat{w}\rangle-\rho_{1}\langle\hat{W}, w\rangle .
$$

Exploiting (7.8) once more and appealing to Lemmas 7.2 and 7.5, the modulus of the right-hand side above is less than or equal to

$$
\begin{aligned}
& c\|u\|_{\mathcal{H}}\left\|w_{x}-l \varphi\right\|+\frac{c}{|\lambda|}\|u\|_{\mathcal{H}}\left\|\varphi_{x}+\psi+l w\right\|+c\|u\|_{\mathcal{H}}\|\xi\|+c\left[\frac{1}{|\lambda|}+1\right]\|u\|_{\mathcal{H}}\|\widehat{u}\|_{\mathcal{H}} \\
& \leq \varepsilon\|u\|_{\mathcal{H}}^{2}+\frac{c}{\varepsilon|\lambda|^{2}}\left\|\varphi_{x}+\psi+l w\right\|^{2}+\frac{c}{\varepsilon}\left[\left\|w_{x}-l \varphi\right\|^{2}+\|\xi\|^{2}\right]+c\left[\frac{1}{|\lambda|}+1\right]\|u\|_{\mathcal{H}}\|\widehat{u}\|_{\mathcal{H}} \\
& \leq c \varepsilon\left[\frac{1}{|\lambda|^{2}}+1\right]\|u\|_{\mathcal{H}}^{2}+\frac{c}{\varepsilon|\lambda|^{2}}\left\|\varphi_{x}+\psi+l w\right\|^{2}+\frac{c}{\varepsilon^{3}}\left[\frac{1}{|\lambda|}+1\right]\|u\|_{\mathcal{H}}\|\widehat{u}\|_{\mathcal{H}},
\end{aligned}
$$

for every $\varepsilon \in(0,1)$ and every $\lambda \neq 0$. The proof is finished.

We now establish two bounds on the term $\varphi_{x}+\psi+l w$. The first bound will be used in the proof of Theorem 4.2, while the second one will be used in the proof of Theorem 4.1.

Lemma 7.7. We have the following estimates.

(i) For every $\varepsilon \in(0,1)$ and every $\lambda \neq 0$ the inequality

$$
k\left\|\varphi_{x}+\psi+l w\right\|^{2} \leq c \varepsilon\left[\frac{1}{|\lambda|^{2}}+1\right]\|u\|_{\mathcal{H}}^{2}+\frac{c}{\varepsilon^{3}}\left[\frac{1}{|\lambda|}+|\lambda|^{2}\right]\left[\|\Psi\|^{2}+\|u\|_{\mathcal{H}}\|\hat{u}\|_{\mathcal{H}}\right]
$$

holds for some structural constant $c>0$ independent of $\varepsilon$ and $\lambda$.

(ii) Assume that $\chi_{g}=0$. Then for every $\varepsilon \in(0,1)$ and every $|\lambda| \geq 1$ the inequality

$$
k\left\|\varphi_{x}+\psi+l w\right\|^{2} \leq c \varepsilon\|u\|_{\mathcal{H}}^{2}+\frac{c}{\varepsilon^{3}}\left[\|\Psi\|^{2}+\|u\|_{\mathcal{H}}\|\hat{u}\|_{\mathcal{H}}\right]
$$

holds for some structural constant $c>0$ independent of $\varepsilon$ and $\lambda$.

Proof. Multiplying (7.7) by $\varphi_{x}+\psi+l w$ in $L^{2}$, we obtain

$$
\begin{aligned}
k\left\|\varphi_{x}+\psi+l w\right\|^{2}= & -\mathrm{i} \lambda \rho_{2}\left\langle\Psi, \varphi_{x}+\psi+l w\right\rangle-b\left\langle\psi_{x},\left(\varphi_{x}+\psi+l w\right)_{x}\right\rangle \\
& +\gamma\left\langle\vartheta,\left(\varphi_{x}+\psi+l w\right)_{x}\right\rangle+\rho_{2}\left\langle\hat{\Psi}, \varphi_{x}+\psi+l w\right\rangle .
\end{aligned}
$$

In the light of (7.4), (7.6) and (7.8), the first term in the right-hand side can be rewritten as

$$
-\mathrm{i} \lambda \rho_{2}\left\langle\Psi, \varphi_{x}+\psi+l w\right\rangle=\rho_{2}\left\langle\Psi, \Phi_{x}\right\rangle+\rho_{2}\|\Psi\|^{2}+l \rho_{2}\langle\Psi, W\rangle+\rho_{2}\left\langle\Psi, \hat{\varphi}_{x}+\hat{\psi}+l \hat{w}\right\rangle .
$$


Appealing to (7.5) and (7.6), we also write the second term in the right-hand side of (7.19) as

$$
\begin{aligned}
-b\left\langle\psi_{x},\left(\varphi_{x}+\psi+l w\right)_{x}\right\rangle= & -\frac{b \rho_{1}}{k}\left\langle\Psi, \Phi_{x}\right\rangle+\frac{b l k_{0}}{k}\left\langle\psi_{x}, w_{x}-l \varphi\right\rangle \\
& -\frac{b l \gamma}{k}\left\langle\psi_{x}, \xi\right\rangle+\frac{b \rho_{1}}{k}\left[\left\langle\psi_{x}, \hat{\Phi}\right\rangle+\left\langle\hat{\psi}_{x}, \Phi\right\rangle\right] .
\end{aligned}
$$

Substituting the two identities above into (7.19), we are led to

$$
k\left\|\varphi_{x}+\psi+l w\right\|^{2}=\gamma\left\langle\vartheta,\left(\varphi_{x}+\psi+l w\right)_{x}\right\rangle+\left(\rho_{2}-\frac{b \rho_{1}}{k}\right)\left\langle\Psi, \Phi_{x}\right\rangle+R_{1},
$$

where

$$
\begin{aligned}
R_{1}= & \rho_{2}\|\Psi\|^{2}+l \rho_{2}\langle\Psi, W\rangle+\frac{b l k_{0}}{k}\left\langle\psi_{x}, w_{x}-l \varphi\right\rangle-\frac{b l \gamma}{k}\left\langle\psi_{x}, \xi\right\rangle \\
& +\rho_{2}\left[\left\langle\hat{\Psi}, \varphi_{x}+\psi+l w\right\rangle+\left\langle\Psi, \hat{\varphi}_{x}+\hat{\psi}+l \hat{w}\right\rangle\right]+\frac{b \rho_{1}}{k}\left[\left\langle\psi_{x}, \hat{\Phi}\right\rangle+\left\langle\hat{\psi}_{x}, \Phi\right\rangle\right]
\end{aligned}
$$

Exploiting now (7.5) and (7.10), we find the equality

$$
\begin{aligned}
\gamma\left\langle\vartheta,\left(\varphi_{x}+\psi+l w\right)_{x}\right\rangle= & \frac{\varpi \rho_{1} \gamma}{\rho_{3} k}\langle\eta, \Phi\rangle_{\mathcal{M}}-\frac{\rho_{1} \gamma^{2}}{\rho_{3} k}\left\langle\Psi, \Phi_{x}\right\rangle+\frac{l \gamma^{2}}{k}\langle\vartheta, \xi\rangle \\
& -\frac{l k_{0} \gamma}{k}\left\langle\vartheta, w_{x}-l \varphi\right\rangle-\frac{\gamma \rho_{1}}{k}[\langle\vartheta, \hat{\Phi}\rangle+\langle\hat{\vartheta}, \Phi\rangle] .
\end{aligned}
$$

Plugging (7.21) into (7.20) and recalling the definition of $R_{1}$, we readily derive the control

$$
k\left\|\varphi_{x}+\psi+l w\right\|^{2} \leq c\left\|\Phi_{x}\right\|\left[\|\Psi\|+\|\eta\|_{\mathcal{M}}\right]+c\|u\|_{\mathcal{H}}\left[\|\Psi\|+\left\|\psi_{x}\right\|+\|\vartheta\|+\|\hat{u}\|_{\mathcal{H}}\right] .
$$

With the aid of (7.4), we see that

$$
\left\|\Phi_{x}\right\| \leq c|\lambda|\left\|\varphi_{x}\right\|+c\left\|\hat{\varphi}_{x}\right\| \leq c|\lambda|\|u\|_{\mathcal{H}}+c\|\hat{u}\|_{\mathcal{H}} .
$$

The estimate above, together with (7.2) and Lemmas 7.1 and 7.3, yield the following bound

$$
\begin{aligned}
& \left\|\Phi_{x}\right\|\left[\|\Psi\|+\|\eta\|_{\mathcal{M}}\right]+\|u\|_{\mathcal{H}}\left[\|\Psi\|+\left\|\psi_{x}\right\|+\|\vartheta\|+\|\hat{u}\|_{\mathcal{H}}\right] \\
& \leq c|\lambda|\|u\|_{\mathcal{H}}\|\Psi\|+c|\lambda|\|u\|_{\mathcal{H}}\|\eta\|_{\mathcal{M}}+c\|u\|_{\mathcal{H}}\left[\|\Psi\|+\left\|\psi_{x}\right\|+\|\vartheta\|\right]+c\|u\|_{\mathcal{H}}\|\hat{u}\|_{\mathcal{H}} \\
& \leq \varepsilon\|u\|_{\mathcal{H}}^{2}+\frac{c}{\varepsilon}\left[\|\Psi\|^{2}+\left\|\psi_{x}\right\|^{2}+\|\vartheta\|^{2}+\|u\|_{\mathcal{H}}\|\hat{u}\|_{\mathcal{H}}\right]+\frac{c|\lambda|^{2}}{\varepsilon}\left[\|\Psi\|^{2}+\|u\|_{\mathcal{H}}\left\|_{\hat{u}}\right\|_{\mathcal{H}}\right] \\
& \leq c \varepsilon\left[\frac{1}{|\lambda|^{2}}+1\right]\|u\|_{\mathcal{H}}^{2}+\frac{c}{\varepsilon^{3}}\left[\frac{1}{|\lambda|}+|\lambda|^{2}\right]\left[\|\Psi\|^{2}+\|u\|_{\mathcal{H}}\|\hat{u}\|_{\mathcal{H}}\right],
\end{aligned}
$$

for every $\varepsilon \in(0,1)$ and every $\lambda \neq 0$. The proof of item (i) is finished.

We now proceed with the proof of item (ii). To this end, we multiply (7.11) by $\varphi$ in $\mathcal{M}$. Invoking (7.4), we infer that

$$
\begin{aligned}
\langle\eta, \Phi\rangle_{\mathcal{M}} & =-\langle\vartheta, \varphi\rangle_{\mathcal{M}}-\langle T \eta, \varphi\rangle_{\mathcal{M}}-\langle\hat{\eta}, \varphi\rangle_{\mathcal{M}}-\langle\eta, \hat{\varphi}\rangle_{\mathcal{M}} \\
& =-g(0)\left\langle\vartheta_{x}, \varphi_{x}\right\rangle-\int_{0}^{\infty} \mu^{\prime}(s)\left\langle\eta_{x}(s), \varphi_{x}\right\rangle \mathrm{d} s-\langle\hat{\eta}, \varphi\rangle_{\mathcal{M}}-\langle\eta, \hat{\varphi}\rangle_{\mathcal{M}},
\end{aligned}
$$

where the second equality follows by integrating by parts in $s$ the term $\langle T \eta, \varphi\rangle_{\mathcal{M}}$. Thus, we get

$$
\langle\eta, \Phi\rangle_{\mathcal{M}}=g(0)\left\langle\vartheta,\left(\varphi_{x}+\psi+l w\right)_{x}\right\rangle-g(0)\left\langle\vartheta, \psi_{x}\right\rangle-l g(0)\left\langle\vartheta, w_{x}\right\rangle
$$




$$
-\int_{0}^{\infty} \mu^{\prime}(s)\left\langle\eta_{x}(s), \varphi_{x}\right\rangle \mathrm{d} s-\langle\hat{\eta}, \varphi\rangle_{\mathcal{M}}-\langle\eta, \hat{\varphi}\rangle_{\mathcal{M}}
$$

Substituting the identity above into (7.21), we obtain

$$
\gamma\left(\varpi g(0)-\frac{\rho_{3} k}{\rho_{1}}\right)\left\langle\vartheta,\left(\varphi_{x}+\psi+l w\right)_{x}\right\rangle=\gamma^{2}\left\langle\Psi, \Phi_{x}\right\rangle+R_{2},
$$

having set

$$
\begin{aligned}
R_{2}= & \varpi \gamma\left[g(0)\left\langle\vartheta, \psi_{x}\right\rangle+l g(0)\left\langle\vartheta, w_{x}\right\rangle+\int_{0}^{\infty} \mu^{\prime}(s)\left\langle\eta_{x}(s), \varphi_{x}\right\rangle \mathrm{d} s\right]-\frac{l \gamma^{2} \rho_{3}}{\rho_{1}}\langle\vartheta, \xi\rangle \\
& +\frac{l k_{0} \gamma \rho_{3}}{\rho_{1}}\left\langle\vartheta, w_{x}-l \varphi\right\rangle+\gamma \rho_{3}[\langle\vartheta, \hat{\Phi}\rangle+\langle\hat{\vartheta}, \Phi\rangle]+\varpi \gamma\left[\langle\hat{\eta}, \varphi\rangle_{\mathcal{M}}+\langle\eta, \hat{\varphi}\rangle_{\mathcal{M}}\right] .
\end{aligned}
$$

At this point, introducing the number

$$
\sigma_{g}=\varpi g(0)-\frac{\rho_{3} k}{\rho_{1}}
$$

and noting that $\chi_{g}=0 \Rightarrow \sigma_{g} \neq 0$, we get

$$
\gamma\left\langle\vartheta,\left(\varphi_{x}+\psi+l w\right)_{x}\right\rangle=\frac{\gamma^{2}}{\sigma_{g}}\left\langle\Psi, \Phi_{x}\right\rangle+\frac{1}{\sigma_{g}} R_{2} .
$$

Plugging this equality into (7.20), we end up with

$$
k\left\|\varphi_{x}+\psi+l w\right\|^{2}=\frac{\varpi g(0) \chi_{g}}{\sigma_{g}}\left\langle\Psi, \Phi_{x}\right\rangle+R_{1}+\frac{1}{\sigma_{g}} R_{2} .
$$

Since $\chi_{g}=0$ by assumption, the first term in the right-hand side vanishes. Hence, recalling the definitions of $R_{1}, R_{2}$ and exploiting (7.1) together with Lemmas 7.1 and 7.3, we estimate

$$
\begin{aligned}
k\left\|\varphi_{x}+\psi+l w\right\|^{2} & \leq c\|u\|_{\mathcal{H}}\left[\|\Psi\|+\left\|\psi_{x}\right\|+\|\vartheta\|+\sqrt{\Gamma[\eta]}+\|\hat{u}\|_{\mathcal{H}}\right] \\
& \leq \varepsilon\|u\|_{\mathcal{H}}^{2}+\frac{c}{\varepsilon}\left[\|\Psi\|^{2}+\left\|\psi_{x}\right\|^{2}+\|\vartheta\|^{2}+\|u\|_{\mathcal{H}}\|\hat{u}\|_{\mathcal{H}}\right] \\
& \leq c \varepsilon\|u\|_{\mathcal{H}}^{2}+\frac{c}{\varepsilon^{3}}\left[\|\Psi\|^{2}+\|u\|_{\mathcal{H}}\|\hat{u}\|_{\mathcal{H}}\right]
\end{aligned}
$$

for every $\varepsilon \in(0,1)$ and every $|\lambda| \geq 1$. The proof is finished.

In the proof of Theorem 4.1 a further bound on the term $\varphi_{x}+\psi+l w$ will be needed.

Lemma 7.8. Assume that $\chi_{h}=0$. Then for every $\varepsilon \in(0,1)$ and every $|\lambda| \geq 1$ the inequality

$$
k\left\|\varphi_{x}+\psi+l w\right\|^{2} \leq c \varepsilon\|u\|_{\mathcal{H}}^{2}+\frac{c}{\varepsilon^{3}}\left[\|W\|^{2}+\|u\|_{\mathcal{H}}\|\hat{u}\|_{\mathcal{H}}\right]
$$

holds for some structural constant $c>0$ independent of $\varepsilon$ and $\lambda$.

Proof. Multiplying (7.9) by $\varphi_{x}+\psi+l w$ in $L^{2}$, we obtain

$$
\begin{aligned}
l k\left\|\varphi_{x}+\psi+l w\right\|^{2}= & -\mathrm{i} \lambda \rho_{1}\left\langle W, \varphi_{x}+\psi+l w\right\rangle-k_{0}\left\langle w_{x}-l \varphi,\left(\varphi_{x}+\psi+l w\right)_{x}\right\rangle \\
& +\gamma\left\langle\xi,\left(\varphi_{x}+\psi+l w\right)_{x}\right\rangle+\rho_{1}\left\langle\hat{W}, \varphi_{x}+\psi+l w\right\rangle .
\end{aligned}
$$

Appealing to (7.4), (7.6) and (7.8), we rewrite the first term in the right-hand side as

$$
-\mathrm{i} \lambda \rho_{1}\left\langle W, \varphi_{x}+\psi+l w\right\rangle=\rho_{1}\left\langle W, \Phi_{x}\right\rangle+\rho_{1}\langle W, \Psi\rangle+l \rho_{1}\|W\|^{2}+\rho_{1}\left\langle W, \hat{\varphi}_{x}+\hat{\psi}+l \hat{w}\right\rangle .
$$


In addition, making use of (7.5) together with (7.4) and (7.8), the second term in the right-hand side of (7.22) can be rewritten as

$$
\begin{aligned}
-k_{0}\left\langle w_{x}-l \varphi,\left(\varphi_{x}+\psi+l w\right)_{x}\right\rangle= & -\frac{k_{0} \rho_{1}}{k}\left\langle W, \Phi_{x}\right\rangle-\frac{l k_{0} \rho_{1}}{k}\|\Phi\|^{2}+\frac{l k_{0}^{2}}{k}\left\|w_{x}-l \varphi\right\|^{2} \\
& -\frac{l k_{0} \gamma}{k}\left\langle w_{x}-l \varphi, \xi\right\rangle+\frac{k_{0} \rho_{1}}{k}\left[\left\langle w_{x}-l \varphi, \hat{\Phi}\right\rangle+\left\langle\hat{w}_{x}-l \hat{\varphi}, \Phi\right\rangle\right] .
\end{aligned}
$$

Plugging the two equalities above into (7.22), we obtain

$$
\begin{aligned}
& l k\left\|\varphi_{x}+\psi+l w\right\|^{2}+\frac{l k_{0} \rho_{1}}{k}\|\Phi\|^{2} \\
& =\gamma\left\langle\xi,\left(\varphi_{x}+\psi+l w\right)_{x}\right\rangle+\left(\rho_{1}-\frac{k_{0} \rho_{1}}{k}\right)\left\langle W, \Phi_{x}\right\rangle+R_{3},
\end{aligned}
$$

having set

$$
\begin{aligned}
R_{3}= & \rho_{1}\langle W, \Psi\rangle+l \rho_{1}\|W\|^{2}+\frac{l k_{0}^{2}}{k}\left\|w_{x}-l \varphi\right\|^{2}-\frac{l k_{0} \gamma}{k}\left\langle w_{x}-l \varphi, \xi\right\rangle \\
& +\rho_{1}\left[\left\langle\hat{W}, \varphi_{x}+\psi+l w\right\rangle+\left\langle W, \hat{\varphi}_{x}+\hat{\psi}+l \hat{w}\right\rangle\right]+\frac{k_{0} \rho_{1}}{k}\left[\left\langle w_{x}-l \varphi, \hat{\Phi}\right\rangle+\left\langle\hat{w}_{x}-l \hat{\varphi}, \Phi\right\rangle\right] .
\end{aligned}
$$

Owing to (7.5) and (7.12), we get

$$
\begin{aligned}
\gamma\left\langle\xi,\left(\varphi_{x}+\psi+l w\right)_{x}\right\rangle= & \frac{\varpi \rho_{1} \gamma}{\rho_{3} k}\langle\zeta, \Phi\rangle_{\mathcal{N}}-\frac{\rho_{1} \gamma^{2}}{\rho_{3} k}\left\langle W, \Phi_{x}\right\rangle-\frac{l \rho_{1} \gamma^{2}}{\rho_{3} k}\|\Phi\|^{2} \\
& +\frac{l \gamma^{2}}{k}\|\xi\|^{2}-\frac{l k_{0} \gamma}{k}\left\langle\xi, w_{x}-l \varphi\right\rangle-\frac{\gamma \rho_{1}}{k}[\langle\xi, \hat{\Phi}\rangle+\langle\hat{\xi}, \Phi\rangle] .
\end{aligned}
$$

Moreover, we multiply (7.13) by $\varphi$ in $\mathcal{N}$. Exploiting (7.4), we are led to

$$
\begin{aligned}
\langle\zeta, \Phi\rangle_{\mathcal{N}} & =-\langle\xi, \varphi\rangle_{\mathcal{N}}-\langle T \zeta, \varphi\rangle_{\mathcal{N}}-\langle\hat{\zeta}, \varphi\rangle_{\mathcal{N}}-\langle\zeta, \hat{\varphi}\rangle_{\mathcal{N}} \\
& =-h(0)\left\langle\xi_{x}, \varphi_{x}\right\rangle-\int_{0}^{\infty} \nu^{\prime}(s)\left\langle\zeta_{x}(s), \varphi_{x}\right\rangle \mathrm{d} s-\langle\hat{\zeta}, \varphi\rangle_{\mathcal{N}}-\langle\zeta, \hat{\varphi}\rangle_{\mathcal{N}}
\end{aligned}
$$

where the second equality follows by integrating by parts in $s$ the term $\langle T \zeta, \varphi\rangle_{\mathcal{N}}$. Thus, we find

$$
\begin{aligned}
\langle\zeta, \Phi\rangle_{\mathcal{N}}= & h(0)\left\langle\xi,\left(\varphi_{x}+\psi+l w\right)_{x}\right\rangle-h(0)\left\langle\xi, \psi_{x}\right\rangle-l h(0)\left\langle\xi, w_{x}\right\rangle \\
& -\int_{0}^{\infty} \nu^{\prime}(s)\left\langle\zeta_{x}(s), \varphi_{x}\right\rangle \mathrm{d} s-\langle\hat{\zeta}, \varphi\rangle_{\mathcal{N}}-\langle\zeta, \hat{\varphi}\rangle_{\mathcal{N}} .
\end{aligned}
$$

A substitution of the identity above into (7.24) yields

$$
\gamma\left(\varpi h(0)-\frac{\rho_{3} k}{\rho_{1}}\right)\left\langle\xi,\left(\varphi_{x}+\psi+l w\right)_{x}\right\rangle=\gamma^{2}\left\langle W, \Phi_{x}\right\rangle+l \gamma^{2}\|\Phi\|^{2}+R_{4},
$$

where

$$
\begin{aligned}
R_{4}= & \varpi \gamma\left[h(0)\left\langle\xi, \psi_{x}\right\rangle+l h(0)\left\langle\xi, w_{x}\right\rangle+\int_{0}^{\infty} \nu^{\prime}(s)\left\langle\zeta_{x}(s), \varphi_{x}\right\rangle \mathrm{d} s\right]-\frac{l \gamma^{2} \rho_{3}}{\rho_{1}}\|\xi\|^{2} \\
& +\frac{l k_{0} \gamma \rho_{3}}{\rho_{1}}\left\langle\xi, w_{x}-l \varphi\right\rangle+\gamma \rho_{3}[\langle\xi, \hat{\Phi}\rangle+\langle\hat{\xi}, \Phi\rangle]+\varpi \gamma\left[\langle\hat{\zeta}, \varphi\rangle_{\mathcal{N}}+\langle\zeta, \hat{\varphi}\rangle_{\mathcal{N}}\right] .
\end{aligned}
$$


At this point, introducing the number

$$
\sigma_{h}=\varpi h(0)-\frac{\rho_{3} k}{\rho_{1}},
$$

and noting that $\chi_{h}=0 \Rightarrow \sigma_{h} \neq 0$, we get

$$
\gamma\left\langle\xi,\left(\varphi_{x}+\psi+l w\right)_{x}\right\rangle=\frac{\gamma^{2}}{\sigma_{h}}\left\langle W, \Phi_{x}\right\rangle+\frac{l \gamma^{2}}{\sigma_{h}}\|\Phi\|^{2}+\frac{1}{\sigma_{h}} R_{4} .
$$

Plugging the equality above into (7.23), we arrive at

$$
l k\left\|\varphi_{x}+\psi+l w\right\|^{2}+\left(\frac{l k_{0} \rho_{1}}{k}-\frac{l \gamma^{2}}{\sigma_{h}}\right)\|\Phi\|^{2}=\frac{\varpi h(0) \chi_{h}}{\sigma_{h}}\left\langle W, \Phi_{x}\right\rangle+R_{3}+\frac{1}{\sigma_{h}} R_{4} .
$$

Being $\chi_{h}=0$ by assumption, the first term in the right-hand side vanishes. For the same reason, we also have

$$
\left(\frac{l k_{0} \rho_{1}}{k}-\frac{l \gamma^{2}}{\sigma_{h}}\right)\|\Phi\|^{2}=l \rho_{1}\|\Phi\|^{2} \geq 0 .
$$

Hence, recalling the definitions of $R_{3}, R_{4}$ and invoking (7.1) together with Lemmas 7.2 and 7.5, we estimate

$$
\begin{aligned}
k\left\|\varphi_{x}+\psi+l w\right\|^{2} & \leq c\|u\|_{\mathcal{H}}\left[\|W\|+\left\|w_{x}-l \varphi\right\|+\|\xi\|+\sqrt{\Gamma[\zeta]}+\|\hat{u}\|_{\mathcal{H}}\right] \\
& \leq \varepsilon\|u\|_{\mathcal{H}}^{2}+\frac{c}{\varepsilon}\left[\|W\|^{2}+\left\|w_{x}-l \varphi\right\|^{2}+\|\xi\|^{2}+\|u\|_{\mathcal{H}}\|\hat{u}\|_{\mathcal{H}}\right] \\
& \leq c \varepsilon\|u\|_{\mathcal{H}}^{2}+\frac{c}{\varepsilon^{3}}\left[\|W\|^{2}+\|u\|_{\mathcal{H}}\|\hat{u}\|_{\mathcal{H}}\right],
\end{aligned}
$$

for every $\varepsilon \in(0,1)$ and every $|\lambda| \geq 1$. The lemma is proved.

Lemma 7.9. For every $\varepsilon \in(0,1)$ and every $\lambda \neq 0$ the inequality

$$
\rho_{1}\|\Phi\|^{2} \leq c \varepsilon\left[\frac{1}{|\lambda|^{2}}+1\right]\|u\|_{\mathcal{H}}^{2}+\frac{c}{\varepsilon^{3}}\left[\frac{1}{|\lambda|}+1\right]\left[\left\|\varphi_{x}+\psi+l w\right\|^{2}+\|u\|_{\mathcal{H}}\|\hat{u}\|_{\mathcal{H}}\right]
$$

holds for some structural constant $c>0$ independent of $\varepsilon$ and $\lambda$.

Proof. Multiplying (7.5) by $\varphi$ in $L^{2}$ and exploiting (7.4), we obtain

$$
\rho_{1}\|\Phi\|^{2}=k\left\langle\varphi_{x}+\psi+l w, \varphi_{x}\right\rangle-l k_{0}\left\langle w_{x}-l \varphi, \varphi\right\rangle+l \gamma\langle\xi, \varphi\rangle-\rho_{1}\langle\hat{\Phi}, \varphi\rangle-\rho_{1}\langle\Phi, \hat{\varphi}\rangle .
$$

In the light of Lemmas 7.2 and 7.5, the modulus of the right-hand side is less than or equal to

$$
\begin{aligned}
& c\|u\|_{\mathcal{H}}\left[\left\|\varphi_{x}+\psi+l w\right\|+\left\|w_{x}-l \varphi\right\|+\|\xi\|+\|\hat{u}\|_{\mathcal{H}}\right] \\
& \leq \varepsilon\|u\|_{\mathcal{H}}^{2}+\frac{c}{\varepsilon}\left[\left\|\varphi_{x}+\psi+l w\right\|^{2}+\left\|w_{x}-l \varphi\right\|^{2}+\|\xi\|^{2}+\|u\|_{\mathcal{H}}\|\hat{u}\|_{\mathcal{H}}\right] \\
& \leq c \varepsilon\left[\frac{1}{|\lambda|^{2}}+1\right]\|u\|_{\mathcal{H}}^{2}+\frac{c}{\varepsilon^{3}}\left[\frac{1}{|\lambda|}+1\right]\left[\left\|\varphi_{x}+\psi+l w\right\|^{2}+\|u\|_{\mathcal{H}}\|\hat{u}\|_{\mathcal{H}}\right]
\end{aligned}
$$

for every $\varepsilon \in(0,1)$ and every $\lambda \neq 0$, and we are finished.

As a first application of the estimates obtained so far, we show that the imaginary axis $i \mathbb{R}$ is contained in the resolvent set $\varrho($ A $)$ of the operator A. Such an inclusion will play a crucial role in the sequel. 
Theorem 7.10. The inclusion $i \mathbb{R} \subset \varrho(\mathrm{A})$ holds.

Proof. Assume by contradiction that $\mathrm{i} \lambda_{0} \in \sigma(\mathrm{A})$ for some $\lambda_{0} \in \mathbb{R}$. Being A the generator of a contraction semigroup, $\mathrm{i} \lambda_{0}$ is necessarily an approximate eigenvalue (cf. [2, Proposition B.2]), meaning that there exists $u_{n}=\left(\varphi_{n}, \Phi_{n}, \psi_{n}, \Psi_{n}, w_{n}, W_{n}, \vartheta_{n}, \eta_{n}, \xi_{n}, \zeta_{n}\right) \in \mathfrak{D}(\mathrm{A})$ satisfying

$$
\left\|u_{n}\right\|_{\mathcal{H}}=1 \quad \text { and } \quad \mathrm{i} \lambda_{0} u_{n}-\mathrm{A} u_{n} \doteq \hat{u}_{n} \rightarrow 0 \quad \text { in } \mathcal{H} \text {. }
$$

Suppose that $\lambda_{0} \neq 0$. Using (7.2)-(7.3) and Lemmas 7.1-7.6, together with Lemma 7.7 item (i) and Lemma 7.9 , for every $\varepsilon>0$ sufficiently small we get the bounds

$$
\begin{aligned}
\left\|u_{n}\right\|_{\mathcal{H}}^{2} & \leq c\left[\frac{1}{\left|\lambda_{0}\right|^{2}}+\left|\lambda_{0}\right|^{2}\right]\left[\varepsilon\left\|u_{n}\right\|_{\mathcal{H}}^{2}+\frac{1}{\varepsilon^{3}}\left[\left\|\varphi_{n x}+\psi_{n}+l w_{n}\right\|^{2}+\left\|\Psi_{n}\right\|^{2}+\left\|u_{n}\right\|_{\mathcal{H}}\left\|\hat{u}_{n}\right\|_{\mathcal{H}}\right]\right] \\
& \leq c\left[\frac{1}{\left|\lambda_{0}\right|^{2}}+\left|\lambda_{0}\right|^{2}\right]^{2}\left[\varepsilon\left\|u_{n}\right\|_{\mathcal{H}}^{2}+\frac{1}{\varepsilon^{15}}\left[\left\|\Psi_{n}\right\|^{2}+\left\|u_{n}\right\|_{\mathcal{H}}\left\|\hat{u}_{n}\right\|_{\mathcal{H}}\right]\right] \\
& \leq c\left[\frac{1}{\left|\lambda_{0}\right|^{2}}+\left|\lambda_{0}\right|^{2}\right]^{3}\left[\varepsilon\left\|u_{n}\right\|_{\mathcal{H}}^{2}+\frac{1}{\varepsilon^{63}}\left\|u_{n}\right\|_{\mathcal{H}}\left\|\hat{u}_{n}\right\|_{\mathcal{H}}\right]
\end{aligned}
$$

where as before $c>0$ denotes a generic constant depending only on the structural quantities of the problem (in particular, independent of $\varepsilon$ and $\lambda_{0}$ ). Fixing $\varepsilon=\varepsilon\left(\lambda_{0}\right)>0$ small enough that

$$
c\left[\frac{1}{\left|\lambda_{0}\right|^{2}}+\left|\lambda_{0}\right|^{2}\right]^{3} \varepsilon<\frac{1}{2},
$$

we conclude that there exists a constant $K=K\left(\lambda_{0}\right)>0$ such that

$$
\left\|u_{n}\right\|_{\mathcal{H}} \leq K\left\|\hat{u}_{n}\right\|_{\mathcal{H}} .
$$

The latter is incompatible with (7.25). We are left to analyze the case $\lambda_{0}=0$. In this situation, relation (7.25) takes the form

$$
\left\|u_{n}\right\|_{\mathcal{H}}=1 \quad \text { and } \quad \mathrm{A} u_{n} \rightarrow 0 \quad \text { in } \mathcal{H} .
$$

Exploiting (4.7), from (7.26) we obtain

$$
\Gamma\left[\eta_{n}\right]+\Gamma\left[\zeta_{n}\right]=\frac{2}{\varpi}\left|\mathfrak{R e}\left\langle\mathrm{A} u_{n}, u_{n}\right\rangle_{\mathcal{H}}\right| \rightarrow 0 .
$$

Due to (4.1)-(4.2), the latter implies that $\eta_{n} \rightarrow 0$ in $\mathcal{M}$ and $\zeta_{n} \rightarrow 0$ in $\mathcal{N}$. Writing the second relation in (7.26) componentwise, we also infer that $\Phi_{n} \rightarrow 0$ in $L^{2}$ and $\Psi_{n}, W_{n} \rightarrow 0$ in $L_{*}^{2}$. Moreover, we get

$$
\begin{aligned}
& k\left(\varphi_{n x}+\psi_{n}+l w_{n}\right)_{x}+l k_{0}\left(w_{n x}-l \varphi_{n}\right)-l \gamma \xi_{n} \rightarrow 0 \text { in } L^{2}, \\
& b \psi_{x x}-k\left(\varphi_{n x}+\psi_{n}+l w_{n}\right)-\gamma \vartheta_{n x} \rightarrow 0 \quad \text { in } L_{*}^{2}, \\
& k_{0}\left(w_{n x}-l \varphi_{n}\right)_{x}-l k\left(\varphi_{n x}+\psi_{n}+l w_{n}\right)-\gamma \xi_{n x} \rightarrow 0 \text { in } L_{*}^{2}, \\
& T \eta_{n}+\vartheta_{n} \rightarrow 0 \text { in } \mathcal{M}, \\
& T \zeta_{n}+\xi_{n} \rightarrow 0 \text { in } \mathcal{N} .
\end{aligned}
$$


Multiplying (7.31) by $\vartheta_{n}$ in the space $\mathcal{M}_{0}=L_{\mu}^{2}\left(\mathbb{R}^{+} ; L^{2}\right)$ and recalling that $\left\|\vartheta_{n}\right\|$ is bounded, we find (cf. the proof of Lemma 7.1)

$$
g(0)\left\|\vartheta_{n}\right\|^{2}+\left\langle T \eta_{n}, \vartheta_{n}\right\rangle_{\mathcal{M}_{0}}=g(0)\left\|\vartheta_{n}\right\|^{2}+\int_{0}^{\infty} \mu^{\prime}(s)\left\langle\eta_{n}(s), \vartheta_{n}\right\rangle \mathrm{d} s \rightarrow 0 .
$$

Invoking (7.27), it is readily seen that $\left|\int_{0}^{\infty} \mu^{\prime}(s)\left\langle\eta_{n}(s), \vartheta_{n}\right\rangle \mathrm{d} s\right| \rightarrow 0$. Thus, $\vartheta_{n} \rightarrow 0$ in $L^{2}$. Making use of (7.32) and arguing in the same way, we also have $\xi_{n} \rightarrow 0$ in $L^{2}$. Finally, we multiply (7.28) by $\varphi_{n},(7.29)$ by $\psi_{n}$ and (7.30) by $w_{n}$ in the respective spaces. Summing up, we obtain the convergence

$$
k\left\|\varphi_{n x}+\psi_{n}+l w_{n}\right\|^{2}+b\left\|\psi_{n x}\right\|^{2}+k_{0}\left\|w_{n x}-l \varphi_{n}\right\|^{2}-\gamma\left\langle\xi_{n}, w_{n x}-l \varphi_{n}\right\rangle-\gamma\left\langle\vartheta_{n}, \psi_{n x}\right\rangle \rightarrow 0 .
$$

Being $\left\|w_{n x}-l \varphi_{n}\right\|,\left\|\psi_{n x}\right\|$ bounded sequences, and recalling that $\left\|\vartheta_{n}\right\|,\left\|\xi_{n}\right\| \rightarrow 0$, the last two terms converge to zero. Hence $\varphi_{n x}+\psi_{n}+l w_{n}$ and $w_{n x}-l \varphi_{n}$ go to zero in $L^{2}$, while $\psi_{n}$ goes to zero in $H_{*}^{1}$. Summarizing, we proved that $\left\|u_{n}\right\|_{\mathcal{H}} \rightarrow 0$, in contradiction with (7.26).

Remark 7.11. The use of approximate eigenvalues (instead of eigenvalues) in the proof above is motivated by the fact that the continuous inclusion $\mathfrak{D}(\mathrm{A}) \subset \mathcal{H}$ is not compact, due to the presence of the memory component (see e.g. [40]). This is also the reason why the estimates given in Lemmas 7.1-7.9 are carried out for every $\lambda \neq 0$ and not only for $|\lambda|$ large enough, in order to perform the contradiction argument for $\lambda_{0} \neq 0$ in the proof of Theorem 7.10.

\section{Proof of ThEOREM 4.1}

The proof is based on the celebrated Gearhart-Prüss theorem [24, 42] (see also [2, Chapter 5] for an historical account).

Theorem 8.1 (Gearhart-Prüss). A bounded semigroup $\Sigma(t)=\mathrm{e}^{t \mathrm{~L}}$ acting on a Hilbert space is exponentially stable if and only if $i \mathbb{R} \subset \varrho(\mathrm{L})$ and

$$
\limsup _{|\lambda| \rightarrow \infty}\left\|(i \lambda-L)^{-1}\right\|<\infty \text {. }
$$

We shall treat the two cases $\chi_{g}=0$ and $\chi_{h}=0$ separately.

$\chi_{g}=0$. We collect (7.2)-(7.3) and Lemmas 7.1-7.6, together with Lemma 7.7 item (ii) and Lemma 7.9. For every $\varepsilon>0$ small enough and every $|\lambda| \geq 1$, we have

$$
\begin{aligned}
\|u\|_{\mathcal{H}}^{2} & \leq c \varepsilon\|u\|_{\mathcal{H}}^{2}+\frac{c}{\varepsilon^{3}}\left[\left\|\varphi_{x}+\psi+l w\right\|^{2}+\|\Psi\|^{2}+\|u\|_{\mathcal{H}}\|\hat{u}\|_{\mathcal{H}}\right] \\
& \leq c \varepsilon\|u\|_{\mathcal{H}}^{2}+\frac{c}{\varepsilon^{15}}\left[\|\Psi\|^{2}+\|u\|_{\mathcal{H}}\|\hat{u}\|_{\mathcal{H}}\right] \\
& \leq c \varepsilon\|u\|_{\mathcal{H}}^{2}+\frac{c}{\varepsilon^{63}}\|u\|_{\mathcal{H}}\|\hat{u}\|_{\mathcal{H}},
\end{aligned}
$$

where the generic structural constant $c>0$ is independent of $\varepsilon$ and $\lambda$. Fixing $\varepsilon>0$ sufficiently small that $c \varepsilon<1 / 2$, there exists a structural constant $K>0$ independent of $\lambda$ such that

$$
\|u\|_{\mathcal{H}} \leq K\|\hat{u}\|_{\mathcal{H}}
$$

for all $|\lambda| \geq 1$. Since Theorem 7.10 tells that $i \mathbb{R} \subset \varrho(A)$, the relation above together with the Gearhart-Prüss theorem yield (4.8). 
$\chi_{h}=0$. We appeal to (7.2)-(7.3), Lemmas 7.1-7.6 and Lemmas 7.8-7.9. For every $\varepsilon>0$ small enough and every $|\lambda| \geq 1$, we have

$$
\begin{aligned}
\|u\|_{\mathcal{H}}^{2} & \leq c \varepsilon\|u\|_{\mathcal{H}}^{2}+\frac{c}{\varepsilon^{3}}\left[\left\|\varphi_{x}+\psi+l w\right\|^{2}+\|W\|^{2}+\|u\|_{\mathcal{H}}\|\hat{u}\|_{\mathcal{H}}\right] \\
& \leq c \varepsilon\|u\|_{\mathcal{H}}^{2}+\frac{c}{\varepsilon^{15}}\left[\|W\|^{2}+\|u\|_{\mathcal{H}}\|\hat{u}\|_{\mathcal{H}}\right] \\
& \leq c \varepsilon\|u\|_{\mathcal{H}}^{2}+\frac{c}{\varepsilon^{31}|\lambda|^{2}}\|u\|_{\mathcal{H}}^{2}+\frac{c}{\varepsilon^{63}}\|u\|_{\mathcal{H}}\|\hat{u}\|_{\mathcal{H}},
\end{aligned}
$$

where again the generic structural constant $c>0$ is independent of $\varepsilon$ and $\lambda$. Fixing $\varepsilon>0$ sufficiently small that $c \varepsilon<1 / 2$, there is a structural constant $K>0$ independent of $\lambda$ such that

$$
\|u\|_{\mathcal{H}}^{2} \leq \frac{K}{|\lambda|^{2}}\|u\|_{\mathcal{H}}^{2}+K\|u\|_{\mathcal{H}}\|\hat{u}\|_{\mathcal{H}}
$$

Therefore, for every $|\lambda| \geq \sqrt{2 K}$, we end up with

$$
\|u\|_{\mathcal{H}} \leq 2 K\|\hat{u}\|_{\mathcal{H}} .
$$

The latter estimate, the inclusion $i \mathbb{R} \subset \varrho(\mathrm{A})$ and the Gearhart-Prüss theorem lead to (4.8).

\section{Proof of Theorem 4.2 - Decay Rate}

The argument relies on the Borichev-Tomilov theorem [4].

Theorem 9.1 (Borichev-Tomilov). Let $\Sigma(t)=\mathrm{e}^{t \mathrm{~L}}$ be a bounded semigroup acting on a Hilbert space, and assume that $\mathrm{i} \mathbb{R} \subset \varrho(\mathrm{L})$. For every fixed $\alpha>0$, we have

$$
\left\|(\mathrm{i} \lambda-\mathrm{L})^{-1}\right\|=\mathrm{O}\left(|\lambda|^{\alpha}\right) \quad \text { as }|\lambda| \rightarrow \infty
$$

if and only if

$$
\left\|\Sigma(t) \mathrm{L}^{-1}\right\|=\mathrm{O}\left(t^{-1 / \alpha}\right) \quad \text { as } t \rightarrow \infty .
$$

We exploit (7.2)-(7.3) and Lemmas 7.1-7.6, together with Lemma 7.7 item (i) and Lemma 7.9. For every $\varepsilon>0$ small enough and $|\lambda| \geq 1$, we have

$$
\begin{aligned}
\|u\|_{\mathcal{H}}^{2} & \leq c \varepsilon\|u\|_{\mathcal{H}}^{2}+\frac{c}{\varepsilon^{3}}\left\|\varphi_{x}+\psi+l w\right\|^{2}+\frac{c|\lambda|^{2}}{\varepsilon^{3}}\left[\|\Psi\|^{2}+\|u\|_{\mathcal{H}}\|\hat{u}\|_{\mathcal{H}}\right] \\
& \leq c \varepsilon\|u\|_{\mathcal{H}}^{2}+\frac{c|\lambda|^{2}}{\varepsilon^{15}}\left[\|\Psi\|^{2}+\|u\|_{\mathcal{H}}\|\hat{u}\|_{\mathcal{H}}\right] \\
& \leq c \varepsilon\|u\|_{\mathcal{H}}^{2}+\frac{c|\lambda|^{2}}{\varepsilon^{63}}\|u\|_{\mathcal{H}}\|\hat{u}\|_{\mathcal{H}}
\end{aligned}
$$

where, as usual, $c>0$ is a generic structural constant independent of $\varepsilon$ and $\lambda$. Fixing $\varepsilon>0$ small enough that $c \varepsilon<1 / 2$, there exists a constant $K>0$ independent of $\lambda$ such that

$$
\|u\|_{\mathcal{H}} \leq K|\lambda|^{2}\|\hat{u}\|_{\mathcal{H}}
$$

for all $|\lambda| \geq 1$. In the light of Theorem 7.10, such a control and the Borichev-Tomilov theorem yield the desired conclusion (4.9). 


\section{LOWER RESOLVENT ESTIMATES FOR THE BGP SYSTEM}

In this section, we establish some lower resolvent estimates that will be the key ingredients in order to prove the second part of Theorem 4.2, namely, the optimality of the decay rate. The following quantitative version of the Riemann-Lebesgue lemma established in [18] will play a crucial role.

Lemma 10.1. Let $f: \mathbb{R}^{+} \rightarrow \mathbb{R}$ be a nonincreasing, absolutely continuous and summable function. Denote by

$$
\hat{f}(\lambda)=\int_{0}^{\infty} f(s) \mathrm{e}^{-\mathrm{i} \lambda s} \mathrm{~d} s
$$

its (half) Fourier transform, and assume that $f(s) \rightarrow f_{0} \in \mathbb{R}$ as $s \rightarrow 0$. Then

$$
\lim _{\lambda \rightarrow \infty} \lambda \hat{f}(\lambda)=-\mathrm{i} f_{0}
$$

Remark 10.2. Actually, the main result of [18] is more general and provides appropriate asymptotic controls on $\hat{f}$ for a wide range of functions, possibly unbounded and nonmonotone. The one reported above is a particular case which is enough for our purposes.

Lemma 10.3. Assume that $\chi_{g} \chi_{h} \neq 0$. Then we have (recall that the inclusion $\mathrm{i} \mathbb{R} \subset \varrho(\mathrm{A})$ has been proved in Theorem 7.10 )

$$
\limsup _{\lambda \rightarrow \infty} \lambda^{-2}\left\|(\mathrm{i} \lambda-\mathrm{A})^{-1}\right\|>0 .
$$

Proof. Calling $\omega_{n}=\frac{n \pi}{\ell}$ for every $n \in \mathbb{N}$, we consider the sequence

$$
\widehat{u}_{n}(x)=\left(0, \frac{\sin \omega_{n} x}{\rho_{1}}, 0,0,0,0,0,0,0,0\right) \in \mathcal{H} .
$$

Note that

$$
\left\|\widehat{u}_{n}\right\|_{\mathcal{H}}=\sqrt{\frac{\ell}{2 \rho_{1}}}, \quad \forall n .
$$

Then, we study the resolvent equation

$$
\mathrm{i} \lambda_{n} u_{n}-\mathrm{A} u_{n}=\widehat{u}_{n}
$$

for some sequence of real numbers $\lambda_{n} \rightarrow \infty$ to be suitably chosen later. Due to Theorem 7.10, there exists a unique solution

$$
u_{n}=\left(\varphi_{n}, \Phi_{n}, \psi_{n}, \Psi_{n}, w_{n}, W_{n}, \vartheta_{n}, \eta_{n}, \xi_{n}, \zeta_{n}\right) \in \mathfrak{D}(\mathrm{A}) .
$$

Using the ansatz

$$
\begin{aligned}
\varphi_{n}(x) & =A_{n} \sin \omega_{n} x, \\
\psi_{n}(x) & =B_{n} \cos \omega_{n} x, \\
w_{n}(x) & =C_{n} \cos \omega_{n} x, \\
\vartheta_{n}(x) & =D_{n} \sin \omega_{n} x, \\
\eta_{n}(x, s) & =d_{n}(s) \sin \omega_{n} x, \\
\xi_{n}(x) & =E_{n} \sin \omega_{n} x, \\
\zeta_{n}(x, s) & =e_{n}(s) \sin \omega_{n} x,
\end{aligned}
$$


for some complex numbers $A_{n}, B_{n}, C_{n}, D_{n}, E_{n}$, and some complex-valued functions

$$
d_{n}, e_{n} \in H_{\mu}^{1}\left(\mathbb{R}^{+}\right) \quad \text { with } \quad d_{n}(0)=e_{n}(0)=0,
$$

after an elementary computation we get the system

$$
\left\{\begin{array}{l}
p_{1}(n) A_{n}+k \omega_{n} B_{n}+l \omega_{n}\left(k+k_{0}\right) C_{n}+l \gamma E_{n}=1, \\
k \omega_{n} A_{n}+p_{2}(n) B_{n}+k l C_{n}+\gamma \omega_{n} D_{n}=0, \\
l \omega_{n}\left(k+k_{0}\right) A_{n}+k l B_{n}+p_{3}(n) C_{n}+\gamma \omega_{n} E_{n}=0, \\
\mathrm{i} \lambda_{n} \rho_{3} D_{n}+\varpi \omega_{n}^{2} \int_{0}^{\infty} \mu(s) d_{n}(s) \mathrm{d} s-\mathrm{i} \lambda_{n} \omega_{n} \gamma B_{n}=0, \\
\mathrm{i} \lambda_{n} d_{n}(s)+d_{n}^{\prime}(s)-D_{n}=0, \\
\mathrm{i} \lambda_{n} \rho_{3} E_{n}+\varpi \omega_{n}^{2} \int_{0}^{\infty} \nu(s) e_{n}(s) \mathrm{d} s-\mathrm{i} \lambda_{n} \gamma\left(\omega_{n} C_{n}+l A_{n}\right)=0, \\
\mathrm{i} \lambda_{n} e_{n}(s)+e_{n}^{\prime}(s)-E_{n}=0,
\end{array}\right.
$$

having set

$$
\begin{aligned}
& p_{1}(n)=-\rho_{1} \lambda_{n}^{2}+k \omega_{n}^{2}+l^{2} k_{0}, \\
& p_{2}(n)=-\rho_{2} \lambda_{n}^{2}+b \omega_{n}^{2}+k, \\
& p_{3}(n)=-\rho_{1} \lambda_{n}^{2}+k_{0} \omega_{n}^{2}+l^{2} k .
\end{aligned}
$$

Integrating the $5^{\text {th }}$ and the $7^{\text {th }}$ equation, we find (recall that $\left.d_{n}(0)=e_{n}(0)=0\right)$

$$
d_{n}(s)=\frac{D_{n}}{\mathrm{i} \lambda_{n}}\left(1-\mathrm{e}^{-\mathrm{i} \lambda_{n} s}\right) \quad \text { and } \quad e_{n}(s)=\frac{E_{n}}{\mathrm{i} \lambda_{n}}\left(1-\mathrm{e}^{-\mathrm{i} \lambda_{n} s}\right) .
$$

Substituting these identities into (10.2), we arrive at

$$
\left\{\begin{array}{l}
p_{1}(n) A_{n}+k \omega_{n} B_{n}+l \omega_{n}\left(k+k_{0}\right) C_{n}+l \gamma E_{n}=1, \\
k \omega_{n} A_{n}+p_{2}(n) B_{n}+k l C_{n}+\gamma \omega_{n} D_{n}=0, \\
l \omega_{n}\left(k+k_{0}\right) A_{n}+k l B_{n}+p_{3}(n) C_{n}+\gamma \omega_{n} E_{n}=0, \\
\lambda_{n}^{2} \omega_{n} \gamma B_{n}+\left[p_{4}(n)-\varpi \omega_{n}^{2} \hat{\mu}\left(\lambda_{n}\right)\right] D_{n}=0, \\
\lambda_{n}^{2} \gamma l A_{n}+\lambda_{n}^{2} \omega_{n} \gamma C_{n}+\left[p_{5}(n)-\varpi \omega_{n}^{2} \hat{\nu}\left(\lambda_{n}\right)\right] E_{n}=0,
\end{array}\right.
$$

having set

$$
\begin{aligned}
& p_{4}(n)=-\rho_{3} \lambda_{n}^{2}+\varpi g(0) \omega_{n}^{2}, \\
& p_{5}(n)=-\rho_{3} \lambda_{n}^{2}+\varpi h(0) \omega_{n}^{2},
\end{aligned}
$$

and (cf. Lemma 10.1)

$$
\hat{\mu}\left(\lambda_{n}\right)=\int_{0}^{\infty} \mu(s) \mathrm{e}^{-\mathrm{i} \lambda_{n} s} \mathrm{~d} s \quad \text { and } \quad \hat{\nu}\left(\lambda_{n}\right)=\int_{0}^{\infty} \nu(s) \mathrm{e}^{-\mathrm{i} \lambda_{n} s} \mathrm{~d} s .
$$


For future use, we introduce the coefficient matrix $\mathbb{M}_{n}$ associated to system (10.3), that is

$$
\mathbb{M}_{n}=\left[\begin{array}{ccccc}
p_{1}(n) & k \omega_{n} & l \omega_{n}\left(k+k_{0}\right) & 0 & l \gamma \\
k \omega_{n} & p_{2}(n) & k l & \gamma \omega_{n} & 0 \\
l \omega_{n}\left(k+k_{0}\right) & k l & p_{3}(n) & 0 & \gamma \omega_{n} \\
0 & \lambda_{n}^{2} \omega_{n} \gamma & 0 & {\left[p_{4}(n)-\varpi \omega_{n}^{2} \hat{\mu}\left(\lambda_{n}\right)\right]} & 0 \\
\lambda_{n}^{2} \gamma l & 0 & \lambda_{n}^{2} \omega_{n} \gamma & 0 & {\left[p_{5}(n)-\varpi \omega_{n}^{2} \hat{\nu}\left(\lambda_{n}\right)\right]}
\end{array}\right] .
$$

We will also need the matrix $\mathbb{A}_{n}$ defined as

$$
\mathbb{A}_{n}=\left[\begin{array}{ccccc}
1 & k \omega_{n} & l \omega_{n}\left(k+k_{0}\right) & 0 & l \gamma \\
0 & p_{2}(n) & k l & \gamma \omega_{n} & 0 \\
0 & k l & p_{3}(n) & 0 & \gamma \omega_{n} \\
0 & \lambda_{n}^{2} \omega_{n} \gamma & 0 & {\left[p_{4}(n)-\varpi \omega_{n}^{2} \hat{\mu}\left(\lambda_{n}\right)\right]} & 0 \\
0 & 0 & \lambda_{n}^{2} \omega_{n} \gamma & 0 & {\left[p_{5}(n)-\varpi \omega_{n}^{2} \hat{\nu}\left(\lambda_{n}\right)\right]}
\end{array}\right] .
$$

Once these preliminary maneuvers are done, for $c_{0} \in \mathbb{R}$ to be suitably fixed later we take

$$
\lambda_{n}=\sqrt{\frac{k \omega_{n}^{2}+l^{2} k_{0}-c_{0}}{\rho_{1}}}=\sqrt{\frac{k}{\rho_{1}}} \omega_{n}+\mathrm{o}\left(\omega_{n}\right) .
$$

With this choice, it is immediate to check that

$$
\begin{aligned}
& p_{1}(n)=c_{0}, \\
& p_{2}(n)=\left(b-\frac{\rho_{2} k}{\rho_{1}}\right) \omega_{n}^{2}+\mathrm{O}(1), \\
& p_{3}(n)=\left(k_{0}-k\right) \omega_{n}^{2}+\mathrm{O}(1), \\
& p_{4}(n)=\left(\varpi g(0)-\frac{\rho_{3} k}{\rho_{1}}\right) \omega_{n}^{2}+\mathrm{O}(1), \\
& p_{5}(n)=\left(\varpi h(0)-\frac{\rho_{3} k}{\rho_{1}}\right) \omega_{n}^{2}+\mathrm{O}(1) .
\end{aligned}
$$

Moreover, Lemma 10.1 (which is applicable due to the assumptions on $\mu$ and $\nu$ ) tells that

$$
\begin{aligned}
& \hat{\mu}\left(\lambda_{n}\right)=-\frac{\mathrm{i} \mu(0)}{\lambda_{n}}+\mathrm{o}\left(\frac{1}{\lambda_{n}}\right), \\
& \hat{\nu}\left(\lambda_{n}\right)=-\frac{\mathrm{i} \nu(0)}{\lambda_{n}}+\mathrm{o}\left(\frac{1}{\lambda_{n}}\right) .
\end{aligned}
$$

Hence, denoting by (cf. the proofs of Lemmas 7.7-7.8)

$$
\sigma_{g}=\varpi g(0)-\frac{\rho_{3} k}{\rho_{1}} \quad \text { and } \quad \sigma_{h}=\varpi h(0)-\frac{\rho_{3} k}{\rho_{1}},
$$


and exploiting (10.4)-(10.11), after a long but elementary computation we find the identity

$$
\operatorname{Det} \mathbb{M}_{n}=\left(\frac{\varpi k}{\rho_{1}}\right) \alpha \omega_{n}^{8}-\mathrm{i} \varpi \sqrt{\frac{\rho_{1}}{k}} \beta \omega_{n}^{7}+\mathrm{o}\left(\omega_{n}^{7}\right),
$$

having set

$$
\alpha=c_{0} \chi_{g} \chi_{h}\left(\frac{\varpi k}{\rho_{1}}\right) g(0) h(0)+\chi_{h} \sigma_{g} k^{2} h(0)+\frac{\chi_{g}}{\rho_{1}}\left[\sigma_{h} \rho_{1}\left(k+k_{0}\right)^{2}-\gamma^{2} k\left(3 k+k_{0}\right)\right] l^{2} g(0)
$$

and

$$
\begin{aligned}
\beta= & c_{0}\left(\frac{\varpi k}{\rho_{1}}\right)\left[h(0) \mu(0)\left(b-\frac{\rho_{2} k}{\rho_{1}}\right) \chi_{h}+g(0) \nu(0)\left(k_{0}-k\right) \chi_{g}\right]-\left(\frac{\varpi k^{3}}{\rho_{1}}\right) h(0) \mu(0) \chi_{h} \\
& -\left(\frac{\varpi k}{\rho_{1}}\right) g(0) \nu(0) l^{2}\left(k+k_{0}\right)^{2} \chi_{g}+\sigma_{g}\left(k_{0}-k\right) k^{2} \nu(0) \\
& +\left(b-\frac{\rho_{2} k}{\rho_{1}}\right) \frac{\left[\sigma_{h} \rho_{1}\left(k+k_{0}\right)^{2}-\gamma^{2} k\left(3 k+k_{0}\right)\right] l^{2} \mu(0)}{\rho_{1}} .
\end{aligned}
$$

Similarly, we also obtain the equality

$$
\operatorname{Det} \mathbb{A}_{n}=\chi_{g} \chi_{h}\left(\frac{\varpi k}{\rho_{1}}\right)^{2} g(0) h(0) \omega_{n}^{8}+\mathrm{o}\left(\omega_{n}^{8}\right)
$$

At this point, we choose $c_{0}$ such that $\alpha=0$, namely

$$
c_{0}=-\frac{1}{\chi_{g} \chi_{h}}\left(\frac{\rho_{1}}{\varpi k}\right) \frac{1}{g(0) h(0)}\left[\chi_{h} \sigma_{g} k^{2} h(0)+\frac{\chi_{g}}{\rho_{1}}\left[\sigma_{h} \rho_{1}\left(k+k_{0}\right)^{2}-\gamma^{2} k\left(3 k+k_{0}\right)\right] l^{2} g(0)\right]
$$

(recall that $\chi_{g} \chi_{h} \neq 0$ by assumption). Substituting the expression of $c_{0}$ into $\beta$, after a few elementary calculations we infer that

$$
\beta=-\frac{\gamma^{2} k^{3}}{\rho_{1} \chi_{g} \chi_{h}}\left[\frac{\mu(0) h(0) \chi_{h}^{2}}{g(0)}+\frac{4 l^{2} \nu(0) g(0) \chi_{g}^{2}}{h(0)}\right] \doteq \beta_{0} \neq 0 .
$$

Therefore, (10.12) turns into

$$
\text { Det } \mathbb{M}_{n}=-\mathrm{i} \varpi \sqrt{\frac{\rho_{1}}{k}} \beta_{0} \omega_{n}^{7}+\mathrm{o}\left(\omega_{n}^{7}\right)
$$

In particular, Det $\mathbb{M}_{n} \neq 0$ for every $n$ sufficiently large, meaning that system (10.3) has a unique solution $\left(A_{n}, B_{n}, C_{n}, D_{n}, E_{n}\right)$. In particular, using Cramer's rule and invoking (10.13)-(10.14) together with (10.4), we learn that

$$
\left|A_{n}\right|=\left|\frac{\operatorname{Det} \mathbb{A}_{n}}{\operatorname{Det} \mathbb{M}_{n}}\right| \sim \mathfrak{c}_{*} \lambda_{n} \quad \text { where } \quad \mathfrak{c}_{*}=\left(\frac{k}{\rho_{1}}\right)^{2} \varpi g(0) h(0)\left|\frac{\chi_{g} \chi_{h}}{\beta_{0}}\right|>0 .
$$

Hence, appealing to (4.4), we derive the controls

$$
\left\|u_{n}\right\|_{\mathcal{H}} \geq \mathfrak{c}\left\|\varphi_{n x}\right\|=\mathfrak{c} \sqrt{\frac{\ell}{2}}\left|A_{n}\right| \omega_{n} \sim \mathfrak{c c}_{*} \sqrt{\frac{\ell \rho_{1}}{2 k}} \lambda_{n}^{2} .
$$

Due to (10.1), we end up with

$$
\limsup _{n \rightarrow \infty} \lambda_{n}^{-2}\left\|\left(\mathrm{i} \lambda_{n}-\mathrm{A}\right)^{-1}\right\| \geq \frac{\mathfrak{c c}_{*} \rho_{1}}{2 \sqrt{k}}>0 .
$$

The proof is finished. 


\section{Proof of Theorem 4.2 - Optimality}

The proof makes use of the Batty-Duyckaerts theorem [3] (see also [2, Theorem 4.4.14]).

Theorem 11.1 (Batty-Duyckaerts). Let $\Sigma(t)=\mathrm{e}^{t \mathrm{~L}}$ be a bounded semigroup acting on a Banach space with $0 \in \varrho(\mathrm{L})$. Assume that $\left\|\Sigma(t) \mathrm{L}^{-1}\right\| \rightarrow 0$ as $t \rightarrow \infty$ and let $d:[0, \infty) \rightarrow \mathbb{R}^{+}$be a decreasing continuous function vanishing at infinity such that $\left\|\Sigma(t) \mathrm{L}^{-1}\right\| \leq d(t)$ for all $t \geq 0$. Then $\mathrm{i} \mathbb{R} \subset \varrho(\mathrm{L})$ and there exists $C>0$ such that

$$
\left\|(\mathrm{i} \lambda-\mathrm{L})^{-1}\right\| \leq C d^{-1}\left(\frac{1}{2|\lambda|}\right)
$$

for all $|\lambda|$ sufficiently large.

Let $\chi_{g} \chi_{h} \neq 0$ and assume by contradiction that (4.10) is not satisfied, namely

$$
\left\|S(t) \mathrm{A}^{-1}\right\|=\mathrm{o}\left(t^{-\frac{1}{2}}\right) \quad \text { as } t \rightarrow \infty .
$$

Take any decreasing continuous function $d:[0, \infty) \rightarrow \mathbb{R}^{+}$such that $\left\|S(t) \mathrm{A}^{-1}\right\| \leq d(t)$ and $d(t)=\mathrm{o}\left(t^{-\frac{1}{2}}\right)$. Exploiting the Batty-Duyckaerts theorem, we learn that

$$
\left\|(\mathrm{i} \lambda-\mathrm{A})^{-1}\right\| \leq C d^{-1}\left(\frac{1}{2|\lambda|}\right)
$$

for some $C>0$ and every $|\lambda|$ large enough. Since

$$
d^{-1}\left(\frac{1}{2|\lambda|}\right)=\mathrm{o}\left(|\lambda|^{2}\right) \quad \text { as }|\lambda| \rightarrow \infty,
$$

we conclude that

$$
\left\|(\mathrm{i} \lambda-\mathrm{A})^{-1}\right\|=\mathrm{o}\left(|\lambda|^{2}\right) \quad \text { as }|\lambda| \rightarrow \infty
$$

in contradiction with Lemma 10.3.

\section{Proof of Theorem 5.1}

We shall prove the two assertions contained in Theorem 5.1 (i.e. the decay rate $\sqrt{t}$ and the optimality) separately. Recall that the inclusion $i \mathbb{R} \subset \varrho(\mathrm{B})$ has been proved in [17].

12.1. Decay rate. We begin by considering the semigroup $S(t)=\mathrm{e}^{t \mathrm{~A}}: \mathcal{H} \rightarrow \mathcal{H}$ generated by equation (4.6) with the particular choice

$$
\mu(s)=\frac{1}{(\varpi \varsigma)^{2}} \mathrm{e}^{-\frac{s}{\varpi \varsigma}} \quad \text { and } \quad \nu(s)=\frac{1}{(\varpi \tau)^{2}} \mathrm{e}^{-\frac{s}{\varpi \tau}}
$$

(note that $\mu$ and $\nu$ fulfill the structural conditions stated in Subsection 4.1 with $g=g_{\varsigma}$ and $h=h_{\tau}$, where $g_{\varsigma}$ and $h_{\tau}$ are defined in (2.1)). Then, in the same spirit of [19, Section 8], we introduce the map $\Lambda: \mathcal{M} \rightarrow L_{*}^{2}$ such that

$$
\Lambda \eta=-\frac{1}{\varpi \varsigma^{2}} \int_{0}^{\infty} \mathrm{e}^{-\frac{s}{\varpi \varsigma}} \eta_{x}(s) \mathrm{d} s,
$$

and the map (denoted with the same symbol) $\Lambda: \mathcal{N} \rightarrow L_{*}^{2}$ such that

$$
\Lambda \zeta=-\frac{1}{\varpi \tau^{2}} \int_{0}^{\infty} \mathrm{e}^{-\frac{s}{\varpi \tau}} \zeta_{x}(s) \mathrm{d} s .
$$


It is readily seen that

$$
\varsigma\|\Lambda \eta\|^{2} \leq \varpi\|\eta\|_{\mathcal{M}}^{2} \quad \text { and } \quad \tau\|\Lambda \zeta\|^{2} \leq \varpi\|\zeta\|_{\mathcal{N}}^{2} .
$$

For every given $u=(\varphi, \Phi, \psi, \Psi, w, W, \vartheta, \eta, \xi, \zeta) \in \mathcal{H}$, we denote by

$$
u^{\Lambda}=(\varphi, \Phi, \psi, \Psi, w, W, \vartheta, \Lambda \eta, \xi, \Lambda \zeta) \in \mathcal{V} .
$$

Let now $v_{0}=\left(\varphi_{0}, \Phi_{0}, \psi_{0}, \Psi_{0}, w_{0}, W_{0}, \vartheta_{0}, p_{0}, \xi_{0}, q_{0}\right) \in \mathfrak{D}(\mathrm{B})$ be an arbitrarily fixed initial datum. Calling for $s>0$

$$
\eta_{0}(x, s)=-\frac{s}{\varpi} \int_{0}^{x} p_{0}(y) \mathrm{d} y \quad \text { and } \quad \zeta_{0}(x, s)=-\frac{s}{\varpi} \int_{0}^{x} q_{0}(y) \mathrm{d} y,
$$

we claim that

$$
\left\{\begin{array}{l}
u_{0} \doteq\left(\varphi_{0}, \Phi_{0}, \psi_{0}, \Psi_{0}, w_{0}, W_{0}, \vartheta_{0}, \eta_{0}, \xi_{0}, \zeta_{0}\right) \in \mathfrak{D}(\mathrm{A}), \\
u_{0}^{\Lambda}=v_{0}
\end{array}\right.
$$

To this end, we need to prove that $\eta_{0}, \zeta_{0} \in \mathfrak{D}(T), \int_{0}^{\infty} \mu(s) \eta_{0}(s) \mathrm{d} s, \int_{0}^{\infty} \nu(s) \zeta_{0}(s) \mathrm{d} s \in H^{2}$ and

$$
\Lambda \eta_{0}=p_{0}, \quad \Lambda \zeta_{0}=q_{0} .
$$

We show the statements for $\eta_{0}$ (the ones for $\zeta_{0}$ are analogous). Note first that $\eta_{0} \in H_{0}^{1}$ for every fixed $s$, since $p_{0} \in L_{*}^{2}$. Next, recalling (12.1), we have

$$
\begin{aligned}
& \int_{0}^{\infty} \mu(s)\left\|\eta_{0 x}(s)\right\|^{2} \mathrm{~d} s=\frac{2 \varsigma}{\varpi}\left\|p_{0}\right\|^{2}<\infty, \\
& \int_{0}^{\infty} \mu(s)\left\|\eta_{0 x}^{\prime}(s)\right\|^{2} \mathrm{~d} s=\frac{1}{\varpi^{3} \varsigma}\left\|p_{0}\right\|^{2}<\infty,
\end{aligned}
$$

meaning that both $\eta_{0}, \eta_{0}^{\prime}$ belong to $\mathcal{M}$. It is also apparent to see that $\left\|\eta_{0 x}(s)\right\| \rightarrow 0$ as $s \rightarrow 0$, yielding $\eta_{0} \in \mathfrak{D}(T)$. The condition $\int_{0}^{\infty} \mu(s) \eta_{0}(s) \mathrm{d} s \in H^{2}$ follows easily from the fact that $p_{0} \in H^{1}$ and $s \mu(s) \in L^{1}\left(\mathbb{R}^{+}\right)$. Finally, we note that

$$
\Lambda \eta_{0}=\frac{p_{0}}{(\varpi \varsigma)^{2}} \int_{0}^{\infty} s \mathrm{e}^{-\frac{s}{\varpi \varsigma}} \mathrm{d} s=p_{0},
$$

and (12.3) is proved.

At this point, using (12.1) and (12.3), one can readily check that $\left[S(t) u_{0}\right]^{\Lambda}$ is the unique (classical) solution to (5.1) with initial datum $v_{0}$, namely

$$
T(t) v_{0}=\left[S(t) u_{0}\right]^{\Lambda} \quad \forall t \geq 0 .
$$

As a consequence, invoking (12.2) and Theorem 4.2, we obtain

$$
\left\|T(t) v_{0}\right\|_{\mathcal{V}}=\left\|\left[S(t) u_{0}\right]^{\Lambda}\right\|_{\mathcal{V}} \leq\left\|S(t) u_{0}\right\|_{\mathcal{H}} \leq \frac{K}{\sqrt{t}}\left\|\mathrm{~A} u_{0}\right\|_{\mathcal{H}}
$$

for some constant $K>0$ and every $t>0$. Exploiting again (12.1) and (12.3), it is also straightforward to check that

$$
\left\|\mathrm{A} u_{0}\right\|_{\mathcal{H}}=\left\|\mathrm{B} v_{0}\right\|_{\mathcal{V}}
$$

Therefore, we end up with

$$
\left\|T(t) v_{0}\right\|_{\mathcal{V}} \leq \frac{K}{\sqrt{t}}\left\|\mathrm{~B} v_{0}\right\|_{\mathcal{V}}
$$

for every $v_{0} \in \mathfrak{D}(\mathrm{B})$ and every $t>0$, and (5.2) is reached. 
12.2. Optimality. Our goal is to show that

$$
\limsup _{\lambda \rightarrow \infty} \lambda^{-2}\left\|(\mathrm{i} \lambda-\mathrm{B})^{-1}\right\|>0
$$

provided that $\chi_{\varsigma} \chi_{\tau} \neq 0$. Once this relation has been proved, (5.3) follows exploiting the BattyDuyckaerts theorem as in Section 11.

Analogously to the proof of Lemma 10.3, we consider the sequence

$$
\widehat{v}_{n}(x)=\left(0, \frac{\sin \omega_{n} x}{\rho_{1}}, 0,0,0,0,0,0,0,0\right) \in \mathcal{V}
$$

where $\omega_{n}=\frac{n \pi}{\ell}$. We have $\left\|\widehat{v}_{n}\right\|_{\mathcal{V}}=\sqrt{\frac{\ell}{2 \rho_{1}}}$ for all $n \in \mathbb{N}$. Then, we study the resolvent equation

$$
\mathrm{i} \lambda_{n} v_{n}-\mathrm{B} v_{n}=\widehat{v}_{n}
$$

for a real sequence $\lambda_{n} \rightarrow \infty$ to be chosen later. The inclusion $i \mathbb{R} \subset \varrho(\mathrm{B})$ tells that such an equation admits a unique solution

$$
v_{n}=\left(\varphi_{n}, \Phi_{n}, \psi_{n}, \Psi_{n}, w_{n}, W_{n}, \vartheta_{n}, p_{n}, \xi_{n}, q_{n}\right) \in \mathfrak{D}(\mathrm{B}) .
$$

Using the ansatz

$$
\begin{aligned}
& \varphi_{n}(x)=A_{n} \sin \omega_{n} x, \\
& \psi_{n}(x)=B_{n} \cos \omega_{n} x, \\
& w_{n}(x)=C_{n} \cos \omega_{n} x, \\
& \vartheta_{n}(x)=D_{n} \sin \omega_{n} x, \\
& p_{n}(x)=\tilde{D}_{n} \cos \omega_{n} x, \\
& \xi_{n}(x)=E_{n} \sin \omega_{n} x, \\
& q_{n}(x)=\tilde{E}_{n} \cos \omega_{n} x,
\end{aligned}
$$

for some complex numbers $A_{n}, B_{n}, C_{n}, D_{n}, \tilde{D}_{n}, E_{n}, \tilde{E}_{n}$, after an elementary calculation we get

$$
\left\{\begin{array}{l}
p_{1}(n) A_{n}+k \omega_{n} B_{n}+l \omega_{n}\left(k+k_{0}\right) C_{n}+l \gamma E_{n}=1, \\
k \omega_{n} A_{n}+p_{2}(n) B_{n}+k l C_{n}+\gamma \omega_{n} D_{n}=0, \\
l \omega_{n}\left(k+k_{0}\right) A_{n}+k l B_{n}+p_{3}(n) C_{n}+\gamma \omega_{n} E_{n}=0, \\
\mathrm{i} \lambda_{n} \rho_{3} D_{n}-\omega_{n} \tilde{D}_{n}-\mathrm{i} \lambda_{n} \omega_{n} \gamma B_{n}=0, \\
\mathrm{i} \lambda_{n} \varsigma \varpi \tilde{D}_{n}+\tilde{D}_{n}+\varpi \omega_{n} D_{n}=0, \\
\mathrm{i} \lambda_{n} \rho_{3} E_{n}-\omega_{n} \tilde{E}_{n}-\mathrm{i} \lambda_{n} \gamma\left(\omega_{n} C_{n}+l A_{n}\right)=0, \\
\mathrm{i} \lambda_{n} \tau \varpi \tilde{E}_{n}+\tilde{E}_{n}+\varpi \omega_{n} E_{n}=0,
\end{array}\right.
$$

where $p_{1}(n), p_{2}(n), p_{3}(n)$ are defined as in the proof of Lemma 10.3. From the $5^{\text {th }}$ and the $7^{\text {th }}$ equation we get

$$
\tilde{D}_{n}=-\frac{\varpi \omega_{n} D_{n}}{\mathrm{i} \lambda_{n} \varsigma \varpi+1} \quad \text { and } \quad \tilde{E}_{n}=-\frac{\varpi \omega_{n} E_{n}}{\mathrm{i} \lambda_{n} \tau \varpi+1}
$$


Accordingly, we arrive at

$$
\left\{\begin{array}{l}
p_{1}(n) A_{n}+k \omega_{n} B_{n}+l \omega_{n}\left(k+k_{0}\right) C_{n}+l \gamma E_{n}=1, \\
k \omega_{n} A_{n}+p_{2}(n) B_{n}+k l C_{n}+\gamma \omega_{n} D_{n}=0 \\
l \omega_{n}\left(k+k_{0}\right) A_{n}+k l B_{n}+p_{3}(n) C_{n}+\gamma \omega_{n} E_{n}=0 \\
\lambda_{n}^{2} \omega_{n} \gamma B_{n}+\left[q_{1}(n)-\frac{\omega_{n}^{2}}{\varsigma\left(\mathrm{i} \lambda_{n} \varsigma \varpi+1\right)}\right] D_{n}=0 \\
\lambda_{n}^{2} \gamma l A_{n}+\lambda_{n}^{2} \omega_{n} \gamma C_{n}+\left[q_{2}(n)-\frac{\omega_{n}^{2}}{\tau\left(\mathrm{i} \lambda_{n} \tau \varpi+1\right)}\right] E_{n}=0
\end{array}\right.
$$

where

$$
q_{1}(n)=-\rho_{3} \lambda_{n}^{2}+\frac{\omega_{n}^{2}}{\varsigma} \quad \text { and } \quad q_{2}(n)=-\rho_{3} \lambda_{n}^{2}+\frac{\omega_{n}^{2}}{\tau} .
$$

The system above is exactly the particular instance of (10.3) corresponding to the choice (12.1). Indeed, in such a case, $q_{1}(n)=p_{4}(n)$ and $q_{2}(n)=p_{5}(n)$, while the Fourier transforms $\hat{\mu}\left(\lambda_{n}\right)$ and $\hat{\nu}\left(\lambda_{n}\right)$ read

$$
\hat{\mu}\left(\lambda_{n}\right)=\frac{1}{\varpi \varsigma\left(\mathrm{i} \lambda_{n} \varsigma \varpi+1\right)} \quad \text { and } \quad \hat{\nu}\left(\lambda_{n}\right)=\frac{1}{\varpi \tau\left(\mathrm{i} \lambda_{n} \tau \varpi+1\right)} .
$$

Hence, the computations in the proof of Lemma 10.3 apply. In particular, recalling that in this situation $\chi_{g}=\chi_{\varsigma}$ and $\chi_{h}=\chi_{\tau}$, relation (12.4) holds provided that $\chi_{\varsigma} \chi_{\tau} \neq 0$.

Remark 12.1. As anticipated in Section 2, making use of the maps $\Lambda$ introduced in Subsection 12.1 and arguing as in [19, Section 8], one can prove that the semigroup $S(t)$ with the particular choice (12.1) is exponentially stable if and only if the same does $T(t)$. We refrain from doing so in this paper, leaving the details to the interested reader.

\section{ProOF OF THEOREM 6.1}

As in the previous section, we shall show the two assertions of Theorem 6.1 separately.

13.1. Decay rate. According to [19], the operator C satisfies the identity

$$
\mathfrak{R e}\langle\mathrm{C} z, z\rangle_{\mathcal{Z}}=-\frac{\varpi}{2} \Gamma[\eta] \leq 0
$$

for all $z \in \mathfrak{D}(\mathrm{C})$, where as before we denote by

$$
\Gamma[\eta]=\int_{0}^{\infty}-\mu^{\prime}(s)\left\|\eta_{x}(s)\right\|^{2} \mathrm{~d} s .
$$

Next, for every fixed $\lambda \in \mathbb{R}$ and $\widehat{z} \in \mathcal{Z}$, we analyze the resolvent equation

$$
\mathrm{i} \lambda z-\mathrm{C} z=\widehat{z}
$$

where $z=(\varphi, \Phi, \psi, \Psi, \vartheta, \eta) \in \mathfrak{D}(\mathrm{C})$. Multiplying such an equation by $z$ in $\mathcal{Z}$, taking the real part and invoking (13.1), we find

$$
\frac{\varpi}{2} \Gamma[\eta]=\mathfrak{R e}\langle\mathrm{i} \lambda z-\mathrm{C} z, z\rangle_{\mathcal{Z}}=\mathfrak{R e}\langle\widehat{z}, z\rangle_{\mathcal{Z}}
$$


Hence, appealing to (4.1), we get

$$
\varpi\|\eta\|_{\mathcal{M}}^{2} \leq c\|z\|_{\mathcal{Z}}\|\widehat{z}\|_{\mathcal{Z}}
$$

for some structural constant $c>0$ independent of $\lambda$. The next lemma summarizes the needed bounds on the other variables of $z$.

Lemma 13.1. For every $\varepsilon>0$ small enough and every $\lambda \neq 0$ the inequalities

$$
\begin{aligned}
& \rho_{3}\|\vartheta\|^{2} \leq \varepsilon\|\Psi\|^{2}+\frac{c}{\varepsilon}\|z\|_{\mathcal{Z}}\|\widehat{z}\|_{\mathcal{Z}}, \\
& b\left\|\psi_{x}\right\|^{2} \leq \frac{\varepsilon}{|\lambda|^{2}}\|z\|_{\mathcal{Z}}^{2}+c \varepsilon\|\Psi\|^{2}+\frac{c}{\varepsilon}\left[\frac{1}{|\lambda|}+1\right]\|z\|_{\mathcal{Z}}\|\widehat{z}\|_{\mathcal{Z}}, \\
& \rho_{2}\|\Psi\|^{2} \leq \frac{c \varepsilon}{|\lambda|^{2}}\|z\|_{\mathcal{Z}}^{2}+\frac{c}{\varepsilon^{3}}\left[\frac{1}{|\lambda|}+1\right]\|z\|_{\mathcal{Z}}\|\widehat{z}\|_{\mathcal{Z}}, \\
& k\left\|\varphi_{x}+\psi\right\|^{2} \leq \varepsilon\|z\|_{\mathcal{Z}}^{2}+\frac{c}{\varepsilon}\left[1+|\lambda|^{2}\right]\left[\|\Psi\|^{2}+\|z\|_{\mathcal{Z}}\|\hat{z}\|_{\mathcal{Z}}\right], \\
& \rho_{1}\|\Phi\|^{2} \leq \varepsilon\|z\|_{\mathcal{Z}}^{2}+\frac{c}{\varepsilon}\left[\left\|\varphi_{x}+\psi\right\|^{2}+\|z\|_{\mathcal{Z}}\|\hat{z}\|_{\mathcal{Z}}\right]
\end{aligned}
$$

hold for some structural constant $c>0$ independent of $\varepsilon$ and $\lambda$.

Proof. The estimates above can be comfortably achieved revisiting the proofs of Lemma 7.1, Lemmas 7.3-7.4, Lemma 7.7 item (i) and Lemma 7.9 in the limit case $l=0$.

Exploiting (13.2) and Lemma 13.1, for every $\varepsilon>0$ small enough and every $\lambda \neq 0$ we get

$$
\|z\|_{\mathcal{Z}}^{2} \leq c \varepsilon\left[\frac{1}{|\lambda|^{2}}+1\right]\|z\|_{\mathcal{Z}}^{2}+\frac{c}{\varepsilon^{15}}\left[\frac{1}{|\lambda|^{2}}+|\lambda|^{2}\right]\|z\|_{\mathcal{Z}}\|\hat{z}\|_{\mathcal{Z}},
$$

where $c>0$ stands for a generic structural constant independent of $\varepsilon$ and $\lambda$. Using (13.3) and arguing as in the proof of Theorem 7.10, one can show that $i \mathbb{R} \subset \varrho(\mathrm{C})$. The details are left to the reader. Moreover, for every $|\lambda| \geq 1$, estimate (13.3) yields

$$
\|z\|_{\mathcal{Z}}^{2} \leq c \varepsilon\|z\|_{\mathcal{Z}}^{2}+\frac{c|\lambda|^{2}}{\varepsilon^{15}}\|z\|_{\mathcal{Z}}\|\hat{z}\|_{\mathcal{Z}}
$$

Hence, fixing $\varepsilon>0$ sufficiently small that $c \varepsilon<1 / 2$, we end up with

$$
\|z\|_{\mathcal{Z}} \leq K|\lambda|^{2}\|\hat{z}\|_{\mathcal{Z}}
$$

for some constant $K>0$ independent of $\lambda$. As in Section 9, the control above together with the Borichev-Tomilov theorem lead to (6.2).

13.2. Optimality. We basically need to revisit the proof of Lemma 10.3 in the limit case $l=0$. To this end, setting as customary $\omega_{n}=\frac{n \pi}{\ell}$, we introduce the sequence

$$
\widehat{z}_{n}(x)=\left(0, \frac{\sin \omega_{n} x}{\rho_{1}}, 0,0,0,0\right) \in \mathcal{Z},
$$

which satisfies $\left\|\widehat{z}_{n}\right\|_{\mathcal{Z}}=\sqrt{\frac{\ell}{2 \rho_{1}}}$ for all $n$. Assuming $\chi_{g} \neq 0$, we consider the resolvent equation

$$
\mathrm{i} \lambda_{n} z_{n}-\mathrm{C} z_{n}=\widehat{z}_{n}
$$


for a real sequence $\lambda_{n} \rightarrow \infty$ to be chosen later. Since $i \mathbb{R} \subset \varrho(\mathrm{C})$, there exists a unique solution

$$
z_{n}=\left(\varphi_{n}, \Phi_{n}, \psi_{n}, \Psi_{n}, \vartheta_{n}, \eta_{n}\right) \in \mathfrak{D}(\mathrm{C}) .
$$

Making use of the ansatz

$$
\begin{aligned}
\varphi_{n}(x) & =A_{n} \sin \omega_{n} x, \\
\psi_{n}(x) & =B_{n} \cos \omega_{n} x, \\
\vartheta_{n}(x) & =C_{n} \sin \omega_{n} x, \\
\eta_{n}(x, s) & =c_{n}(s) \sin \omega_{n} x,
\end{aligned}
$$

for some complex numbers $A_{n}, B_{n}, C_{n}$ and some complex-valued function $c_{n} \in H_{\mu}^{1}\left(\mathbb{R}^{+}\right)$with $c_{n}(0)=0$, after an elementary calculation we get the system

$$
\left\{\begin{array}{l}
r_{1}(n) A_{n}+k \omega_{n} B_{n}=1, \\
k \omega_{n} A_{n}+r_{2}(n) B_{n}+\gamma \omega_{n} C_{n}=0, \\
\mathrm{i} \lambda_{n} \rho_{3} C_{n}+\varpi \omega_{n}^{2} \int_{0}^{\infty} \mu(s) c_{n}(s) \mathrm{d} s-\mathrm{i} \lambda_{n} \omega_{n} \gamma B_{n}=0 \\
\mathrm{i} \lambda_{n} c_{n}(s)+c_{n}^{\prime}(s)-C_{n}=0
\end{array}\right.
$$

having set

$$
r_{1}(n)=-\rho_{1} \lambda_{n}^{2}+k \omega_{n}^{2} \quad \text { and } \quad r_{2}(n)=-\rho_{2} \lambda_{n}^{2}+b \omega_{n}^{2}+k .
$$

Integrating the last equation of the system above and substituting the resulting expression into the third equation, we arrive at

$$
\left\{\begin{array}{l}
r_{1}(n) A_{n}+k \omega_{n} B_{n}=1 \\
k \omega_{n} A_{n}+r_{2}(n) B_{n}+\gamma \omega_{n} C_{n}=0 \\
\lambda_{n}^{2} \omega_{n} \gamma B_{n}+\left[r_{3}(n)-\varpi \omega_{n}^{2} \hat{\mu}\left(\lambda_{n}\right)\right] C_{n}=0
\end{array}\right.
$$

where

$$
r_{3}(n)=-\rho_{3} \lambda_{n}^{2}+\varpi g(0) \omega_{n}^{2} \quad \text { and } \quad \hat{\mu}\left(\lambda_{n}\right)=\int_{0}^{\infty} \mu(s) \mathrm{e}^{-\mathrm{i} \lambda_{n} s} \mathrm{~d} s .
$$

At this point, we choose

$$
\lambda_{n}=\sqrt{\frac{k \omega_{n}^{2}-c_{0}}{\rho_{1}}}=\sqrt{\frac{k}{\rho_{1}}} \omega_{n}+\mathrm{o}\left(\omega_{n}\right),
$$

for some $c_{0} \in \mathbb{R}$ to be fixed later. In this situation, it is immediate to check that

$$
\begin{aligned}
& r_{1}(n)=c_{0}, \\
& r_{2}(n)=\left(b-\frac{\rho_{2} k}{\rho_{1}}\right) \omega_{n}^{2}+\mathrm{O}(1), \\
& r_{3}(n)=\left(\varpi g(0)-\frac{\rho_{3} k}{\rho_{1}}\right) \omega_{n}^{2}+\mathrm{O}(1), \\
& \hat{\mu}\left(\lambda_{n}\right)=-\frac{\mathrm{i} \mu(0)}{\lambda_{n}}+\mathrm{o}\left(\frac{1}{\lambda_{n}}\right),
\end{aligned}
$$


where the last equality follows from Lemma 10.1. Analogously to the proof of Lemma 10.3, we introduce the coefficient matrix $\mathbb{M}_{n}$ associated to (13.4), that is

$$
\mathbb{M}_{n}=\left[\begin{array}{ccc}
r_{1}(n) & k \omega_{n} & 0 \\
k \omega_{n} & r_{2}(n) & \gamma \omega_{n} \\
0 & \lambda_{n}^{2} \omega_{n} \gamma & {\left[r_{3}(n)-\varpi \omega_{n}^{2} \hat{\mu}\left(\lambda_{n}\right)\right]}
\end{array}\right]
$$

Exploiting the asymptotic relations above, after an elementary computation we find

$$
\operatorname{Det} \mathbb{M}_{n}=\alpha \omega_{n}^{4}-\mathrm{i} \varpi \mu(0) \sqrt{\frac{\rho_{1}}{k}} \beta \omega_{n}^{3}+\mathrm{o}\left(\omega_{n}^{3}\right),
$$

having set

$$
\alpha=-c_{0} \chi_{g} \frac{\varpi g(0) k}{\rho_{1}}-k^{2}\left(\varpi g(0)-\frac{\rho_{3} k}{\rho_{1}}\right) \quad \text { and } \quad \beta=k^{2}-c_{0}\left(b-\frac{k \rho_{2}}{\rho_{1}}\right) .
$$

Similarly, considering the matrix $\mathbb{A}_{n}$ defined as

$$
\mathbb{A}_{n}=\left[\begin{array}{ccc}
1 & k \omega_{n} & 0 \\
0 & r_{2}(n) & \gamma \omega_{n} \\
0 & \lambda_{n}^{2} \omega_{n} \gamma & {\left[r_{3}(n)-\varpi \omega_{n}^{2} \hat{\mu}\left(\lambda_{n}\right)\right]}
\end{array}\right],
$$

we obtain the equality

$$
\operatorname{Det} \mathbb{A}_{n}=-\chi_{g} \frac{\varpi g(0) k}{\rho_{1}} \omega_{n}^{4}+\mathrm{o}\left(\omega_{n}^{4}\right)
$$

Choosing $c_{0}$ such that $\alpha=0$, and then substituting the expression of $c_{0}$ into $\beta$, we obtain (recall that $\chi_{g} \neq 0$ by assumption)

$$
\beta=\frac{\gamma^{2} k^{2}}{\chi_{g} \varpi g(0)} \doteq \beta_{0} \neq 0
$$

Accordingly, (13.5) takes the form

$$
\operatorname{Det} \mathbb{M}_{n}=-\mathrm{i} \varpi \mu(0) \sqrt{\frac{\rho_{1}}{k}} \beta_{0} \omega_{n}^{3}+\mathrm{o}\left(\omega_{n}^{3}\right)
$$

In particular, Det $\mathbb{M}_{n} \neq 0$ for every $n$ sufficiently large and thus system (13.4) has a unique solution $\left(A_{n}, B_{n}, C_{n}\right)$. Using Cramer's rule and invoking (13.6), together with the equality above and the definition of $\lambda_{n}$, we infer that

$$
\left|A_{n}\right|=\left|\frac{\operatorname{Det} \mathbb{A}_{n}}{\operatorname{Det} \mathbb{M}_{n}}\right| \sim \mathfrak{c}_{*} \lambda_{n} \quad \text { where } \quad \mathfrak{c}_{*}=\frac{g(0) k}{\mu(0) \rho_{1}}\left|\frac{\chi_{g}}{\beta_{0}}\right|>0 .
$$

Finally, arguing as in the last part of the proof of Lemma 10.3 (in particular, using the equivalence between $\|\cdot\|_{\mathcal{Z}}$ and the product norm, and the fact that $\left\|\widehat{z}_{n}\right\|_{\mathcal{Z}}$ is constant), we get

$$
\limsup _{n \rightarrow \infty} \lambda_{n}^{-2}\left\|\left(i \lambda_{n}-\mathrm{C}\right)^{-1}\right\|>0 \text {. }
$$

Relation (6.3) now follows exploiting the Batty-Duyckaerts theorem as in Section 11. 


\section{REFERENCES}

[1] F. Alabau Boussouira, J.E. Muñoz Rivera and D.S. Almeida Júnior, Stability to weak dissipative Bresse system, J. Math. Anal. Appl. 374 (2011), 481-498.

[2] W. Arendt, C.J.K. Batty, M. Hieber and F. Neubrander, Vector-valued Laplace transforms and Cauchy problems, Birkhäuser, Basel, 2011.

[3] C.J.K. Batty and T. Duyckaerts, Non-uniform stability for bounded semi-groups on Banach spaces, J. Evol. Equ. 8 (2008), 765-780.

[4] A. Borichev and Y. Tomilov, Optimal polynomial decay of functions and operator semigroups, Math. Ann. 347 (2010), 455-478.

[5] J.A.C. Bresse, Cours de méchanique appliquée, Mallet-Bachelier, Paris, 1859.

[6] C.L. Cardozo, M.A. Jorge Silva, T.F Ma and J.E. Muñoz Rivera, Stability of Timoshenko systems with thermal coupling on the bending moment, Math. Nachr. 292 (2019), 2537-2555.

[7] C. Cattaneo, Sulla conduzione del calore, Atti Semin. Matemat. Univ. Modena 3 (1948), 83-101.

[8] W. Charles, J.A. Soriano, F.A. Falcão Nascimento and J.H. Rodrigues, Decay rates for Bresse system with arbitrary nonlinear localized damping, J. Differential Equations 255 (2013), 2267-2290.

[9] B.D. Coleman and M.E. Gurtin, Equipresence and constitutive equations for rigid heat conductors, Z. Angew. Math. Phys. 18 (1967), 199-208.

[10] M. Conti, V. Pata and M. Squassina, Singular limit of differential systems with memory, Indiana Univ. Math. J. 55 (2006), 170-213.

[11] M. Coti Zelati, F. Dell'Oro and V. Pata, Energy decay of type III linear thermoelastic plates with memory, J. Math. Anal. Appl. 401 (2013), 357-366.

[12] C.M. Dafermos, Asymptotic stability in viscoelasticity, Arch. Rational Mech. Anal. 37 (1970), 297-308.

[13] V. Danese and F. Dell'Oro, The lack of exponential stability for a class of second-order systems with memory, Proc. Roy. Soc. Edinburgh Sect. A 147 (2017), 683-702.

[14] F. Dell'Oro, Asymptotic stability of thermoelastic systems of Bresse type, J. Differential Equations 258 (2015), 3902-3927.

[15] H.D. Fernández Sare and R. Racke, On the stability of damped Timoshenko systems: Cattaneo versus Fourier law, Arch. Rational Mech. Anal. 194 (2009), 221-251.

[16] P.R. de Lima and H.D. Fernández Sare, Stability of thermoelastic Bresse systems, Z. Angew. Math. Phys. 70 (2019), Paper No. 3, 33 pp.

[17] P.R. de Lima and H.D. Fernández Sare, General condition for exponential stability of thermoelastic Bresse systems with Cattaneo's law, Commun. Pure Appl. Anal. 19 (2020), 3575-3596.

[18] F. Dell'Oro, E. Laeng and V. Pata, A quantitative Riemann-Lebesgue lemma with application to equations with memory, Proc. Amer. Math. Soc. 145 (2017), 2909-2915.

[19] F. Dell'Oro and V. Pata, On the stability of Timoshenko systems with Gurtin-Pipkin thermal law, J. Differential Equations 257 (2014), 523-548.

[20] I. Elishakoff, Who developed the so-called Timoshenko beam theory?, Math. Mech. Solids 25 (2020), 97116.

[21] L.H. Fatori, P.R. de Lima and H.D. Fernández Sare, A nonlinear thermoelastic Bresse system: Global existence and exponential stability, J. Math. Anal. Appl. 443 (2016), 1071-1089.

[22] L.H. Fatori and R.N. Monteiro, The optimal decay rate for a weak dissipative Bresse system, Appl. Math. Lett. 25 (2012), 600-604.

[23] L.H. Fatori and J.E. Muñoz Rivera, Rates of decay to weak thermoelastic Bresse system, IMA J. Appl. Math. 75 (2010), 881-904.

[24] L. Gearhart, Spectral theory for contraction semigroups on Hilbert spaces, Trans. Amer. Math. Soc. 236 (1978), 385-394.

[25] M. Grasselli and V. Pata, Uniform attractors of nonautonomous systems with memory, in "Evolution Equations, Semigroups and Functional Analysis" (A. Lorenzi and B. Ruf, Eds.), pp.155-178, Progr. Nonlinear Differential Equations Appl. no.50, Birkhäuser, Boston, 2002.

[26] A. Guesmia and M. Kafini, Bresse system with infinite memories, Math. Methods Appl. Sci. 38 (2015), 23892402. 
[27] A. Guesmia and M. Kirane, Uniform and weak stability of Bresse system with two infinite memories, Z. Angew. Math. Phys. 67 (2016), Art. 124, 39 pp.

[28] M.E. Gurtin and A.C. Pipkin, A general theory of heat conduction with finite wave speeds, Arch. Rational Mech. Anal. 31 (1968), 113-126.

[29] M. Khader and B. Said-Houari, On the decay rate of solutions of the Bresse system with Gurtin-Pipkin thermal law, Asymptot. Anal. 103 (2017), 1-32.

[30] J.E. Lagnese, G. Leugering and E.J.P.G. Schmidt, Modelling of dynamic networks of thin thermoelastic beams, Math. Methods Appl. Sci. 16 (1993), 327-358.

[31] J.E. Lagnese, G. Leugering and E.J.P.G. Schmidt, Modeling, analysis and control of dynamic elastic multilink structures, Birkhäuser, Boston, 1994.

[32] Z. Liu and B. Rao, Energy decay rate of the thermoelastic Bresse system, Z. Angew. Math. Phys. 60 (2009), $54-69$.

[33] Z. Liu and S. Zheng, Semigroups associated with dissipative systems, Chapman \& Hall/CRC, Boca Raton, 1999.

[34] T.F. Ma and R.N. Monteiro, Singular limit and long-time dynamics of Bresse systems, SIAM J. Math. Anal. 49 (2017), 2468-24958.

[35] S. Messaoudi and J.H. Hassan, New general decay results in a finite-memory Bresse system, Commun. Pure Appl. Anal. 18 (2019), 1637-1662.

[36] J.E. Muñoz Rivera and M.G. Naso, Boundary stabilization of Bresse systems, Z. Angew. Math. Phys. 70 (2019), Paper No. 56, 16 pp.

[37] J.E. Muñoz Rivera and R. Racke, Mildly dissipative nonlinear Timoshenko systems-global existence and exponential stability, J. Math. Anal. Appl. 276 (2002), 248-278.

[38] P. Olsson and G. Kristensson, Wave splitting of the Timoshenko beam equation in the time domain, Z. Angew. Math. Phys. 45 (1994), 866-881.

[39] V. Pata and E. Vuk, On the exponential stability of linear thermoelasticity, Contin. Mech. Thermodyn. 12 (2000), 121-130.

[40] V. Pata and A. Zucchi, Attractors for a damped hyperbolic equation with linear memory, Adv. Math. Sci. Appl. 11 (2001), 505-529.

[41] A. Pazy, Semigroups of linear operators and applications to partial differential equations, Springer-Verlag, New York, 1983.

[42] J. Prüss, On the spectrum of $C_{0}$-semigroups, Trans. Amer. Math. Soc. 284 (1984), 847-857.

[43] R. Racke, Thermoelasticity with second sound-exponential stability in linear and non-linear 1-d, Math. Methods Appl. Sci. 25 (2002), 409-441.

[44] R. Racke, Thermoelasticity. Handbook of differential equations: evolutionary equations. Vol. V, 315-420, Elsevier/North-Holland, Amsterdam, 2009.

[45] B. Said-Houari and A. Soufyane, The Bresse system in thermoelasticity, Math. Methods Appl. Sci. 38 (2015), 3642-3652.

[46] M.L. Santos, D.S. Almeida Júnior and J.E. Muñoz Rivera, The stability number of the Timoshenko system with second sound, J. Differential Equations 253 (2012), 2715-2733.

[47] M.L. Santos, A. Soufyane and D.S. Almeida Júnior, Asymptotic behavior to Bresse system with past history, Quart. Appl. Math. 73 (2015), 23-54.

[48] J.A. Soriano, J.E. Muñoz Rivera and L.H. Fatori, Bresse system with indefinite damping, J. Math. Anal. Appl. 387 (2012), 284-290.

[49] B. Straughan, Heat waves, Springer, New York, 2011.

[50] S.P. Timoshenko, On the correction for shear of a differential equation for transverse vibrations of prismatic bars, Philos. Magazine 41 (1921), 744-746.

[51] A. Wehbe and W. Youssef, Exponential and polynomial stability of an elastic Bresse system with two locally distributed feedbacks, J. Math. Phys. 51 (2010), 103523, 17 pp.

Politecnico di Milano - Dipartimento di Matematica

Via BonaRdi 9, 20133 Milano, ItALY

Email address: filippo.delloro@polimi.it 